\title{
Molecular simulation for predicting the rheological properties of polymer melts
}

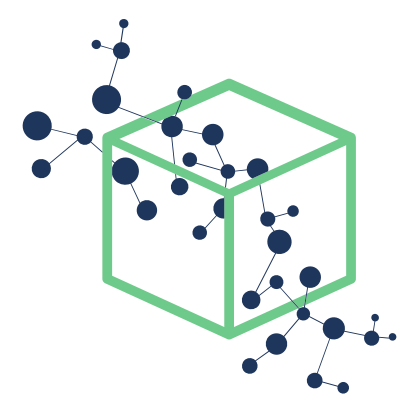

XI RESEARCH

http://xiresearch.org

DOI: $10.1080 / 08927022.2019 .1605600$

This document is the accepted manuscript (after peer review) version of an article published in its final form (i.e., the version of record) by Taylor \& Francis as

Li Xi. Molecular simulation for predicting the rheological properties of polymer melts. Molecular Simulation, 45:1242-1264, 2019. doi: 10.1080/08927022.2019.1605600

(copyright (C 2019, Taylor \& Francis). The version of record is hosted at

https://dx.doi.org/10.1080/08927022.2019.1605600

by the publisher.

The current version is made available under the CC-BY-NC-ND 4.0 license (https://creativecommons. org/licenses/by-nc-nd/4.0/) in accordance with the publisher's policy. Please refer to the publisher's site for additional terms of use.

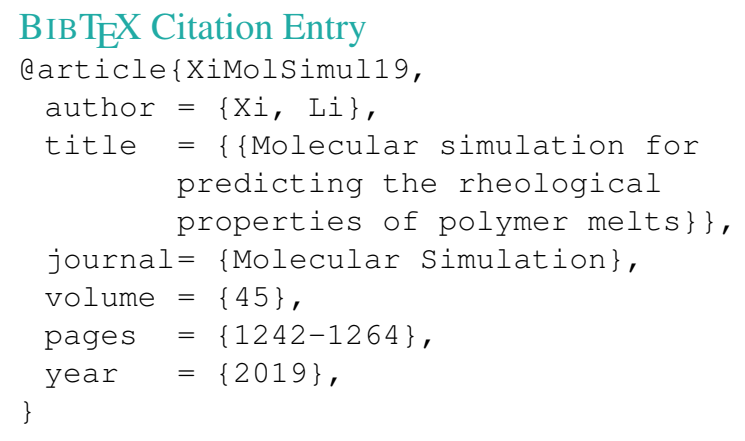

\begin{tabular}{|c|c|c|}
\hline \multicolumn{3}{|r|}{$\begin{array}{c}\text { brought to you by the } \\
\text { XI RESEARCH GROUP at McMaster University }\end{array}$} \\
\hline & & $\begin{array}{c}\text { WwW.XIRESEARCH.org - } \\
\text { Principle Investigator: } \\
\text { Li Xi 奚力 } \\
\text { Email: li@xiresearch.org }\end{array}$ \\
\hline & in & $\begin{array}{l}\text { https://twitter.com/xiresearchgroup } \\
\text { https://linkedin.com/company/xiresearch/ } \\
\text { https://researchgate.net/profile/Li_Xi16 } \\
\text { https://mendeley.com/profiles/li-xi11/ }\end{array}$ \\
\hline
\end{tabular}




\title{
Molecular simulation for predicting the rheological properties of polymer melts
}

\author{
Li Xi \\ Department of Chemical Engineering, McMaster University, Hamilton, Ontario L8S 4L7, \\ Canada
}

\section{ARTICLE HISTORY}

Compiled December 3, 2019

\begin{abstract}
Bottom-up prediction that links materials chemistry to their properties is a constant theme in polymer simulation. Rheological properties are particularly challenging to predict because of the extended time scales involved as well as large uncertainty in the stress output from molecular simulation. This review focuses on the application of molecular simulation in the prediction of such properties, including approaches solely based on molecular simulation and its integration with rheological models. Most attention is given to the prediction of quantitative properties, in particular, those most studied such as shear viscosity and linear viscoelasticity. Studies on the fundamental understanding of rheology are referenced only when they are directly relevant to the property prediction. The review starts with an overview of the major methods for extracting rheological properties from molecular simulation, using bead-spring chain models as a sandbox system. It then discusses materials-specific prediction using chemically-realistic models, including systematically coarse-grained models that allow the mapping between scales. Finally, integrating molecular simulation with rheological models extends the prediction to highly entangled polymers. Recent development of several multiscale predictive frameworks allowed the successful prediction of rheological properties from the chemical structure for polymers of experimentally relevant molecular weights.
\end{abstract}

\section{KEYWORDS}

polymer melts; rheology; viscoelasticity; plateau modulus; dynamic moduli; shear viscosity; molecular simulation; multiscale modeling

\section{Introduction}

Synthetic polymers are ubiquitous in our industrialized society. These materials are cost-effective, light-weight, easy to process, and, most importantly, highly customizable - properties of polymer materials can be easily modified by adjusting their formulations or processing conditions. The richness of polymer behaviors and properties stems from the wide spectrum of length scales in its microstructure. As a result, simple changes in the chemistry of individual monomers, their ordering in the chain sequence (copolymers, tacticity, etc.), connection topology of different pieces of a polymer (linear, branched, etc.), arrangement of chain segments (crystalline vs. amorphous), overall molecular size, and what they are mixed with (blends, composites, etc.) can all lead to drastic and often unpredictable changes in material properties. Take polyethylene for 
example: introduction of a very low density of long-chain branches to linear polyethylene (less than one branch per $2 \times 10^{4}$ backbone carbons or less than one branch per six chains) causes an over six-fold increase in its shear viscosity [1].

Computer simulation plays a vital role in our understanding of the complex dynamics in polymer materials and, ultimately prediction of their properties a priori. (For a general overview of simulation techniques for polymer systems, the reader is referred to review articles by Glotzer \& Paul [2] and Paul \& Smith [3].) The wide span of length and time scales in polymer materials is also the biggest obstacle in their simulation. For instance, nowadays, the duration of a typical atomistic (using models retaining most chemical details, including the chemical structure of monomers) molecular dynamics $(\mathrm{MD})$ simulation run, for a simulation domain of $\approx(5 \mathrm{~nm})^{3}$, falls into the $O(10)-O(100)$ ns range, whereas the dynamics of long-chain polymers can easily reach macroscopic times scales (seconds or minutes).

This obstacle is felt across all aspects of polymer simulation, starting from the generation of a computer representation of a realistic polymer configuration (e.g., as an initial condition for MD). For long-chain polymers, the time scale to fully equilibrate the chain conformation, i.e., the longest polymer relaxation time, is well beyond the reach of brute-force MD. For this reason, the development of methods for polymer structure generation has remained an active area of research after more than three decades [4-9]. For dynamical properties and phenomena, the limited time scale accessible by MD is a more direct challenge. A classical problem widely studied by molecular simulation is the diffusion of small penetrant molecules in an amorphous polymer matrix [10-12]. Even for the smallest gas or liquid molecules, direct prediction of their diffusivity by MD can still be prohibitively expensive, owing to the large time scales of the physical process. Advanced sampling or modeling approaches must be coupled with molecular simulation to overcome the challenge [13-16].

The current review focuses on the rheological properties of polymers. The molecular process behind material rheological responses is polymer relaxation, which, in essence, is the diffusion of polymer chains in a matrix made of other polymer chains. With this perspective, it becomes clear that the problem involves substantially longer time scales than the aforementioned problem of small-molecule diffusion in polymers. For this reason, computing the rheological properties of viscoelastic polymers, even in the case of highly simplified coarse-grained (CG) models, is a daunting task. Moreover, rheological quantities calculated from MD simulation are prone to errors and large uncertainty. For atomistic molecular models, direct prediction of the linear viscoelasticity of long-chain polymers is still beyond reach. Recent advancement in systematic coarse-graining techniques as well as the development of increasingly accurate mesoscopic rheological models offer new solutions to these challenges, which can be used to extend the reach of atomistic molecular simulation. Several such multiscale predictive modeling frameworks have been proposed in recent years. The success seen so far in those efforts brings hope that the reliable rheological prediction of practical polymer materials through computer simulation is getting close to reality.

Note the difference between rheological property prediction (focus of this review) and the development of theories and models in rheology per se - the latter has been the subject of a number of review articles over the years [17-21]. This review concentrates instead on the application of molecular simulation in the ab initio rheological property predictions, either by itself or as part of an integrated predictive framework, and highlights the strengths and limitations of existing approaches. The phrase " $a b$ initio", in this context, describes a systematic bottom-up prediction strategy that uses information from the "very beginning" - i.e., material chemical structure and makeup 
(at the level of atomistic molecular models) - to predict its macroscopic properties that are directly verifiable in experiments. (This is not to be confused to its meaning in " $a b$ initio molecular dynamics" which refers to the direct computation of intermolecular potentials at the quantum mechanics level). This usage of the term is the same as in Padding \& Briels [22]. The review focuses on the prediction of quantitative properties that can, eventually, be directly compared with standard measurements from experimental rheometry. For this reason, much coverage is given to steady-shear rheology and linear viscoelasticity. Progress in the fundamental understanding, such as chain conformation statistics under more complex flow types, is not discussed in detail and only referenced when needed. A more detailed discussion of the review's limited scope is given in section 4 .

The review is organized as follows. Section 2 focuses on approaches based solely on MD. It starts with an overview of the major techniques for extracting rheological properties from MD simulation, using the most-studied bead-spring chain model system as a sandbox case (section 2.1). Major difficulties and challenges in each approach and strategies for their mitigation will also be discussed. Applications of these techniques in the MD simulation of chemically-realistic models - i.e., models that represent specific polymer species - are discussed in section 2.2, starting with force-field based atomistic models (section 2.2.1) where direct MD prediction is only possible for a very limited range of systems. Systematic CG approaches that allow access to larger length and time scales are then discussed in section 2.2.2, with an emphasize on their application in predicting the rheological properties of long-chain polymers. Section 3 extends the discussion to integrated frameworks that combine molecular simulation with rheological models, including the Rouse model for unentangled polymers (section 3.1) and tube and slip-link (slip-spring) models for entangled ones (section 3.2). Invoking rheological models helps to either reduce the uncertainty in the data or project the prediction to longer time scales (for long and highly-entangled chains). The paper is concluded with a summary and outlook discussion in section 4 .

\section{Direct prediction from molecular dynamics}

\subsection{The bead-spring chain model as a sandbox: overview of major MD approaches}

The seminal work by Kremer and Grest [23] established a CG model for a generic polymer (i.e., without specifying its chemical species) which is still widely used to this day. In this model, often referred to as the Kremer-Grest (KG) model in the literature, a polymer chain consists of multiple Lennard-Jones (LJ) particles or beads - pointforce sites interacting with the LJ pairwise potential [24]. Each bead is considered a repeating unit or, loosely-speaking, "monomer" of this model chain. Chemical connectivity between these beads is enforced by the finitely-extensible nonlinear elastic (FENE) spring potential [25]. (Monomers and FENE bonds in the KG model do not map one-to-one to repeating units and chemical bonds in any real polymer. Because of the flexibility of FENE bonds, each CG bead would instead represent a blob containing many realistic chemical repeating units. The FENE bond potential becomes a reasonable model for describing the bead-bead distance statistics only at sufficiently high levels of CG - when each bead represents a chain segment much longer than the Kuhn length of the polymer [26].) Although variations can be introduced to the interparticle potentials to account for the effects of different chemical types (e.g., adding 
angle potentials between consecutive FENE bonds to model the bending rigidity of the chain [27]), this model does not offer any direct representation of the chemical structure and thus cannot make $a b$ initio predictions for specific polymer species. It, however, captures the universal aspects of chain structure such as the length and architecture of the chain. (Such universality is only relevant at scales much larger than that of the Kuhn monomers. At small scales, both chain statics and dynamics are strongly affected by the specific chemical structures, as demonstrated by the recent work of Takahashi et al. [28].) For linear chains, it successfully captures the full range of dynamics from the Rouse dynamics at the short-time limit to - in the case of entangled polymers - reptation at long time [23]. Mutating the bead connection topology can easily adapt the model for other molecular architectures such as branched, crosslinked, and ring polymers [29-32]. Because of its simplicity and lower computational cost, the FENE chain model is often used as a sandbox for the development and proof-of-concept demonstration of simulation techniques. Major approaches for computing rheological quantities from MD were all initially applied to the FENE chains before their adaption to more complex and chemically-realistic models.

\subsubsection{Non-equilibrium molecular dynamics (NEMD) approach}

The most intuitive approach is to construct virtual "experiments" in computer simulation that reflect the actual experimental setup. For shear rheology, a planar Couette flow can be simulated using the SLLOD equations of motion [33] coupled with a boundary condition that properly describes the shear deformation, with the Lees-Edwards sliding-box boundary condition [34] being the most common choice for homogeneous shear flow [35]. Since the early work of Kröger et al. [36], a number of studies have simulated the steady shear flow of FENE chains using NEMD and studied their steady shear viscosity, normal stress coefficients, and flow-induced chain alignment [37-39]. Shear viscosity of the model displays the typical behavior of polymer melts, which starts with a constant magnitude (the Newtonian plateau) at low shear rates $\dot{\gamma}$ and crosses over to a power-law decay

$$
\eta(\dot{\gamma}) \propto \dot{\gamma}^{-n}
$$

(the shear-thinning region) at higher $\dot{\gamma}$. The power-law exponent $n$ varies between studies ( 0.40 to 0.42 for $N=50$ in ref. [37], $0.60 \pm 0.10$ for $N=10$ to 400 in ref. [38], and 0.89 for $N=400$ in ref. [39], $N$ being the degree of polymerization - i.e., the number of beads in a chain) but all predictions fall into the experimentally observed range of 0.4 to 0.9 [40]. The crossover point shifts to lower $\dot{\gamma}$ with higher $N$, which reflects the increasing relaxation time of longer chains. Note that for a given chain length, the time required for the shear stress to reach the steady state decreases with $\dot{\gamma}$ [41]. The computational cost is thus higher at the low $\dot{\gamma}$ end and it becomes increasingly difficult to capture the Newtonian plateau for longer chains [37-39].

Extension to small-amplitude oscillatory shear (SAOS) seems straightforward by imposing a sinusoidal shear strain

$$
\gamma(t)=\gamma_{0} \sin (\omega t)
$$

to the simulation cell, where $\gamma_{0}$ is the strain oscillation amplitude and $\omega$ is the angular frequency. For viscoelastic fluids, the shear stress output time series will oscillate at the same frequency but with a phase shift (to the left - i.e., earlier time) $\delta \in[0, \pi / 2]$ 
( $\delta=0$ and $\pi / 2$ correspond to the purely elastic and viscous limits, respectively).

$$
\begin{aligned}
\sigma_{x y} & =\sigma_{x y, 0} \sin (\omega t+\delta) \\
& =\gamma_{0}\left(G^{\prime} \sin (\omega t)+G^{\prime \prime} \cos (\omega t)\right)
\end{aligned}
$$

where

$$
\begin{aligned}
G^{\prime}(\omega) & \equiv \frac{\sigma_{x y, 0}}{\gamma_{0}} \cos (\delta) \\
G^{\prime \prime}(\omega) & \equiv \frac{\sigma_{x y, 0}}{\gamma_{0}} \sin (\delta)
\end{aligned}
$$

are the storage (elastic) and loss (viscous) modulus, respectively and the phase angle

$$
\delta=\arctan \left(\frac{G^{\prime \prime}}{G^{\prime}}\right)
$$

[42]. For polymers modeled by FENE chains, NEMD oscillatory shear simulation was first performed by Hernández Cifre et al. [43] (for $N=5$ to 70) and then by Vladkov $\&$ Barrat [44] (for $N=10$ and 20). The method was shown to generate robust results that agree well with theoretical predictions (at least in the parameter ranges tested). However, it is also computationally demanding as NEMD simulation needs to be performed for every frequency level and a large number of oscillation periods are required at each frequency for reliable statistics (100 periods at each frequency in ref. [43]). The latter requirement makes it costly to capture the low-frequency (i.e., long period) regime. It is for this reason that results are typically only reported for fairly short chain lengths and relatively small frequency ranges with $\omega$ spanning less than 3 decades. In ref. [43], because $\omega$ only reached $10^{-2}$ (in LJ reduced units [45]) at the lower limit, the terminal scaling of $G^{\prime} \propto \omega^{2}$ and $G^{\prime \prime} \propto \omega$, according to the Rouse model prediction [46], was only observed at the shortest chain length of $N=5$. In addition, since all these chains are below the critical chain length $N_{\mathrm{c}}$ for entanglement (which, for FENE chains, is $O(100)$ but depends on the method of measurement $[38,47,48]$ ), the rubbery plateau of reptation dynamics has not been captured.

Kremer and coworkers [47,49] have used a different NEMD approach that extracts the linear viscoelasticity from a volume-conserving elongational step-strain, i.e.,

$$
\begin{gathered}
L_{x}=\lambda^{-\frac{1}{2}} L \\
L_{y}=\lambda^{-\frac{1}{2}} L \\
L_{z}=\lambda L
\end{gathered}
$$

where $L$ is the original cubic box length at equilibrium. Assuming affine deformation, the stress relaxation modulus, defined as

$$
G(t) \equiv \frac{\sigma_{x y}(t)}{\gamma_{0}}
$$

(where $\sigma_{x y}(t)$ is the transient shear stress response after an infinitesimal step shear strain $\gamma_{0}$ imposed on the equilibrium configuration), can be related to the normal 

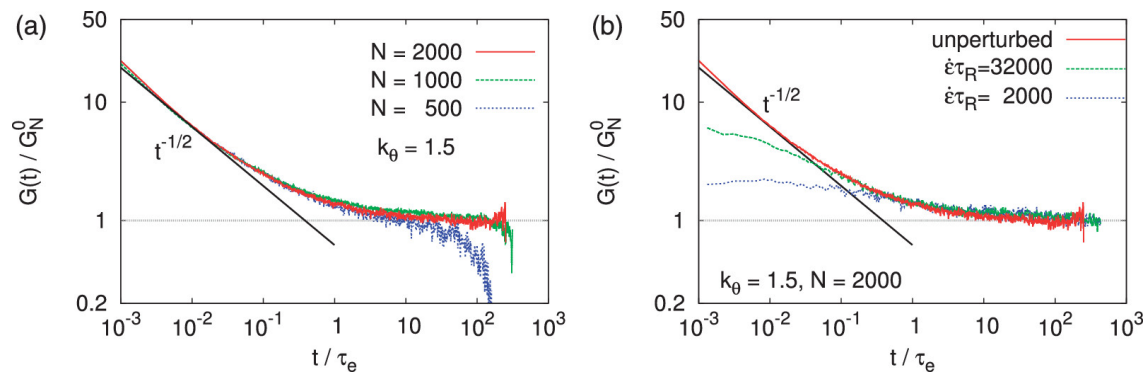

Figure 1. Stress relaxation modulus $G(t)$ scaled by the plateau modulus $G_{N}^{0}$ of semi-flexible FENE bead spring chains (with bending potential imposed for each bond angle $\theta-U_{\text {bend }}(\theta)=k_{\theta}(1-\cos \theta)$ ): (a) results from the Green-Kubo relation of different chain lengths $\mathrm{N}$ and (b) comparison between the Green-Kubo ("unperturbed") and the step-strain elongation NEMD (eq. (11)) approaches ( $\dot{\epsilon}$ is the extension rate; $\tau_{\mathrm{e}}$ and $\tau_{\mathrm{R}}$ are the Rouse relaxation times of an entanglement strand and of the entire chain, respectively). Each simulation cell contains $n_{\text {chain }}=1000$ chains and a moving average over 100 time steps ( 1 LJ time unit) was applied to the $\sigma_{x y}(t)$ signal before the stress autocorrelation function (SAF) calculation. The $t<\tau_{\mathrm{e}}$ region, i.e., relaxation within entanglement strands, shows typical Rouse dynamics with the scaling of $G(t) \propto t^{-1 / 2}$. The plateau modulus (reptation regime) is noticeable at $N=500$ and becomes pronounced at $N=O(1000)$. The terminal relaxation regime for the two higher $N$ is not captured because of the limited time range available. (Reprinted from ref. [49], with permission of AIP Publishing.)

stress output of the step-elongation simulation via

$$
G(t)=\frac{\sigma_{z z}(t)}{\lambda^{2}-\frac{1}{\lambda}}
$$

where $\sigma_{z z}(t)$ is the time-dependent normal stress following the step-strain [42]. The deformation needs to be small to remain in the linear response regime (i.e., $\lambda$ close to unity). Figure 1(b) [49] compares $G(t)$ from this NEMD approach (eq. (11)) with that from the equilibrium MD approach (eq. (12) below, which is rigorously in the linear regime): the finite-size elongational strain causes the $G(t)$ to be underestimated in the Rouse regime: $t<\tau_{\mathrm{e}}$. (The equilibration time $\tau_{\mathrm{e}}$ marks the crossover between the short-time Rouse dynamics within entanglement strands and the long-time reptation dynamics of the whole chains.) For the reptation/plateau regime, results from the two approaches agree excellently. The relaxation modulus contains the full information of the material viscoelasticity in the linear regime, from which the zero-shear-rate viscosity $\eta_{0}$ and dynamic moduli $G^{\prime}$ and $G^{\prime \prime}$ can be calculated (see section 2.1.2 below).

\subsubsection{Equilibrium $M D$ or Green-Kubo approach}

2.1.2.1. Overview of the method. In the limit of linear response, transport properties are related to the time correlation function of the equilibrium flux fluctuations through the Green-Kubo relation [50]. In the case of shear rheology, this means $G(t)$ is proportional to the autocorrelation function of the shear stress

$$
G(t)=\frac{V}{k_{\mathrm{B}} T}\left\langle\sigma_{x y}\left(t_{0}\right) \sigma_{x y}\left(t_{0}+t\right)\right\rangle_{t_{0}}
$$

( $t$ is the time lag, $\langle\cdot\rangle_{t_{0}}$ indicates average over different time origins $t_{0}, k_{\mathrm{B}}$ is the Boltzmann constant, and $V$ and $T$ are the system volume and temperature, respectively). Because of the equilibrium nature of this approach, for shear viscosity, only its zero- 
shear-rate limit value is available [42]

$$
\eta_{0} \equiv \lim _{\dot{\gamma} \rightarrow 0} \eta(\dot{\gamma})=\int_{0}^{\infty} G(t) d t
$$

$\mathrm{Xu}$ et al. [51] used this approach to calculate the zero-shear-rate viscosity of FENE chains with $N=10$ to 50 and the resulting $\eta_{0}$ (from eq. (13)) compares well with the extrapolation of the $\eta(\dot{\gamma})$ profiles (in their earlier study with the NEMD approach [37]) to the $\dot{\gamma} \rightarrow 0$ limit. Excellent agreement was also found between the Green-Kubo result of ref. [52] and the NEMD result of ref. [36].

In addition to $\eta_{0}$, the dynamic moduli $G^{\prime}$ and $G^{\prime \prime}$ can be calculated from $G(t)$ using

$$
\begin{aligned}
G^{\prime}(\omega) & =\omega \int_{0}^{\infty} G(t) \sin (\omega t) d t \\
G^{\prime \prime}(\omega) & =\omega \int_{0}^{\infty} G(t) \cos (\omega t) d t .
\end{aligned}
$$

[42]. Compared with the NEMD approach, the Green-Kubo approach only requires one equilibrium MD (EMD) run from which the dynamic moduli over the entire spectrum of frequency can be calculated. This approach was first adopted by Sen et al. [52] for FENE chains with $N=20$ to 120 , which was followed by a number of studies by different researchers, with recent studies reaching chain length $N$ up to 2000 [49,53-55].

2.1.2.2. Uncertainty and noise caused by stress fluctuations. A well-known difficulty of the Green-Kubo approach is that the obtained $G(t)$ profiles are loaded with strong noises, which, if not properly treated, can often obscure the main signal and, in some cases, lead to incorrect conclusions. The primary cause is the strong stress fluctuations in MD simulation which are intrinsic to any microscopic simulation cell. This is further compounded by the fact that the autocorrelation function of any finite time series always has larger uncertainty at the large-time-lag end, because of the diminishing number of independent sample pairs: in the case of the stress autocorrelation function (SAF) calculation (needed for $G(t)$ via eq. (12)), the number of statistically-independent $\sigma_{x y}\left(t_{0}\right)$ and $\sigma_{x y}\left(t_{0}+t\right)$ pairs decreases with increasing $t$. This poses great challenges for the determination of the plateau modulus $G_{N}^{0}$. Entangled melts of linear polymers are typically very soft and the $G_{N}^{0}$ magnitude is usually much lower than the fluctuations in the $G(t)$ profile: e.g., in the EMD simulation of Likhtman et al. [54], $G_{N}^{0}=O\left(10^{-2}\right), 4$ orders of magnitude lower compared with $G(0)=O(100)$ (note from eq. (12) that $G(0)=\left(V /\left(k_{\mathrm{B}} T\right)\right)\left\langle\sigma_{x y}^{2}\right\rangle$, proportional to the stress fluctuation magnitude squared). The plateau, if existent, occurs at the long time limit where the noise in SAF is largest. It is indeed common to see the $G(t)$ signal buried by noises in that regime. In general, $G_{N}^{0}$ obtained from the Green-Kubo approach, without proper treatment of the noise, is often subject to large uncertainty and errors. Likewise for the entanglement strand length $N_{\mathrm{e}}$, which is often derived from the plateau modulus via

$$
G_{N}^{0}=\frac{4}{5} \frac{\nu k_{\mathrm{B}} T}{N_{\mathrm{e}}}
$$

(where $\nu$ is the monomer number density) according to the tube theory [46]. 
It is for this reason that earlier studies using the Green-Kubo approach often contained unreliable results. The initial work by Sen et al. [52] reported the observation of plateaus in both $G^{\prime}(\omega)$ and $G^{\prime \prime}(\omega)$ profiles for $N \gtrsim 80$. The estimated $N_{\mathrm{e}}$ based on the observed stress plateaus is 28 which is close to $N_{\mathrm{e}} \approx 30$ estimated from $\tau_{\mathrm{e}}$ identified on the segmental mean-square displacement (MSD) curve [47]. It is however at least a factor of 2 lower than the $N \approx 80$ estimate from the plateau modulus measured from the step-strain elongation NEMD simulation [47], which should be closer because both the Green-Kubo and step-stain elongation approaches estimate $N_{\mathrm{e}}$ from $G_{N}^{0}$ - see fig. 1(b). Later studies at similar or even longer chain length, as discussed below, were not able to reproduce these observations. (In addition, Masubuchi \& Uneyama [56] recently compared MD results using the FENE bead-spring chain model from different researchers and found that the chain diffusivity data from Sen et al. [52] do not match those of other studies even for much shorter - unentangled - chains.) For a similar chain length of $N=100$, Lee \& Kremer [55] reported a plateau in the $G(t)$ profile from which the estimated $N_{\mathrm{e}}=96$. This result, again, was later found to be an artifact of the large noise-to-signal ratio and the improper treatment thereof. The reported $G_{N}^{0}$ in Lee \& Kremer [55] was at the same order of magnitude as the fluctuations in the $G(t)$ signal (moving average was applied to the $\sigma_{x y}(t)$ signal before eq. (12) was invoked). It was later shown that after the noise is properly filtered (discussed below), $G(t)$ is still slowly decaying with $t$ in the time range originally identified as the plateau $[57,58]$.

Indeed, Zhou and Larson [53] and Likhtman et al. [54] showed that a well-defined stress relaxation plateau does not appear even for much longer chains. For flexible FENE chains with $N=100$ (close or equal to refs. [52,55]), the relaxation dynamics nearly completely follows the Rouse dynamics. Entanglement effects become obvious for $N \gtrsim 200$ where $G(t)$ deviates from the Rouse scaling of $t^{1 / 2}$ but a strict plateau does not appear for $N$ up to 350 tested in ref. [54]. It was thus concluded that for the level of chain length $(N=O(100)), G_{N}^{0}$ and $N_{\mathrm{e}}$ cannot be directly measured from rheological data alone and theoretical models must be invoked. By fitting the MD results with the slip-spring model [59] (further discussed in section 3.2.2) and calculating its $G_{N}^{0}$ prediction at the $N \rightarrow \infty$ limit, Likhtman et al. [54] estimated $N_{\mathrm{e}}=51.5$, which is closer to but still lower than the step-elongation result of ref. [47]. Likhtman and Sukumaran [57] further predicted that the stress relaxation plateau cannot be clearly identified in $G(t)$ for chain length $N<O(10) N_{\mathrm{e}}$ (which is consistent with experimental observations [60]). For flexible FENE chains, this means $N \gtrsim O(1000)$ would be required for the reliable extraction of $G_{N}^{0}$ from the $G(t)$ profile.

A major hurdle to the direct computation of $G_{N}^{0}$ from MD is thus the difficulty of generating the required amorphous chain structures. Not only does the chain length $N$ has to reach at least $O(1000)$, a large number of these chains must also be packed into the simulation cell to minimize the correlation between the periodic images of the chains. This became possible only recently with the the latest algorithmic developments for efficient amorphous chain equilibration [8,9]. For a semi-flexible FENE bead-spring model, Hsu \& Kremer [49] calculated $G(t)$ using the Green-Kubo approach for $N=500,1000$ and 2000 and all three cases give nearly the same $N_{\mathrm{e}} \approx 28$ (fig. 1(a)). $\left(N_{\mathrm{e}}\right.$ decreases with chain rigidity - thus the lower value here than those quoted above for flexible FENE chains.) For this semi-flexible chain model, a stress plateau is discernible at $N=500\left(>10 N_{\mathrm{e}}\right)$ and can be unambiguously identified for $N>1000$.

2.1.2.3. Noise reduction methods. Through these trials and errors, various tech- 
niques and protocols have been tested for reducing the noise and improving the reliability of the outcome. The ultimate way to reduce the uncertainty in a statistic is to increase the size of the sample. In MD simulation, this means prolonging the simulation run, enlarging the system size (stress fluctuation $\propto N_{\mathrm{p}}^{-1 / 2}$ where $N_{\mathrm{p}}$ is the number of particles in the system), and/or using more independent samples. For the last one, an easy step to take is to average the SAF between all shear components of the stress tensor

$$
G(t)=\frac{V}{3 k_{\mathrm{B}} T}\left(\left\langle\sigma_{x y}(0) \sigma_{x y}(t)\right\rangle+\left\langle\sigma_{x z}(0) \sigma_{x z}(t)\right\rangle+\left\langle\sigma_{y z}(0) \sigma_{y z}(t)\right\rangle\right)
$$

thanks to the isotropy of the EMD system $[49,55]$. This, however, only increases the sample size by a factor of 3 , which alone is not sufficient to address the noise reduction challenge. For increasing simulation time, Vladkov \& Barrat [44] estimated (assuming Gaussian statistics of the stress fluctuation) that reducing the relative uncertainty of the SAF at the large-time-lag limit to a manageable level of $10 \%$ would require a simulation length of $10^{6} \tau_{\mathrm{c}}$, where $\tau_{\mathrm{c}}$ is the correlation time of the $\sigma_{x y}(t)$ signal. If $\tau_{\mathrm{c}}$ equals the longest relaxation time of the - in their case unentangled - chains $\tau_{\mathrm{R}}$ (the Rouse time), the required simulation length would be prohibitive. (The situation would be worse for entangled polymers whose relaxation times are longer than $\tau_{R}$.) This conclusion was challenged by Sen et al. [61] who argued that stress fluctuation is dominated by short-time bond vibrations. As least for a short unentangled melt with $N=80, \tau_{c} \sim 10^{-4} \tau_{\mathrm{R}}$. A much shorter simulation time of $\sim 100 \tau_{\mathrm{R}}$ would thus suffice. Large noise in the SAF function $\left\langle\sigma_{x y}(0) \sigma_{x y}(t)\right\rangle$ was still found at the large-time-lag end which can be effectively reduced by applying a running or moving average filter to the $\left\langle\sigma_{x y}(0) \sigma_{x y}(t)\right\rangle$ signal (with the window size growing linearly with the time lag). Moving average was shown to have no discernible effect on the integral of the SAF (i.e., $\eta_{0}$; see eq. (13)).

Other than filtering the SAF, moving average has also been tested on the original stress signal $\sigma_{x y}(t)$ (which is equivalent to the moving average of the SAF using a triangular window function [62]). This treatment reduces the SAF magnitude at the short time end but does not affect the plateau magnitude for a wide range of average window sizes [55]. (As reviewed above, despite the significant reduction in the noise, the remaining uncertainty in that study was still too large for the correct identification of a stress relaxation plateau or, indeed, the lack thereof $[57,58]$.) For strongly entangled polymers, Hsu \& Kremer [49] were able to produce a clear stress relaxation plateau with a noise level significantly lower than the signal (i.e., $G_{N}^{0}$; see fig. 1(a)) by combing the moving average in $\sigma_{x y}(t)$ with a significantly enlarged system size: a total of $n_{\text {chain }}=1000$ chains with $N$ up to 2000 (compared with $n_{\text {chain }}=100$ and $N=$ 100 in ref. [55]). In addition to averaging over all shear components (eq. (17)), the study also averaged over $O(10)$ independent simulation runs to eliminate the potential dependence on the initial polymer configuration.

Ramírez et al. [62] adapted a multiple-tau correlator algorithm widely used in the processing of dynamic light scattering data [63] to MD simulation applications, which is able to calculate the autocorrelation function of any time series with noise filtering on the fly. (Time correlation without filtering is also possible.) During the MD simulation, the algorithm keeps multiple levels of the time series data (in a "data" array) and the calculated correlation function values (in a "correlation" array). At the first level, the data array stores the original time series for a short time period and the correlation array stores the correlation function at smallest time lags. At higher levels, the data 
array contains moving-averaged time series (window size increases with the level rank) that cover longer time periods, and the correlation array stores the correlation function at larger time lags. The outcome is thus equivalent to applying moving average to the original time series with a hierarchy of window sizes and the correlation function uses moving averages over smaller windows for small time lags and averages over larger windows for larger time lags. For application to the SAF and Green-Kubo approach for $G(t)$, the algorithm has been shown to effectively smoothen the result especially at large time lags - i.e., the entangled regime - with a moderate simulation system size $\left(N \sim O(100)\right.$ and $\left.n_{\text {chain }} \sim O(100)\right)[54,57]$.

Other noise-reduction approaches have been reported in applications using atomistic molecular models, which will be discussed in section 2.2.1. In addition to filtering the MD data during postprocessing, rheological models have also been used to circumvent the stress fluctuation problem, which will be discussed in section 3 .

\subsubsection{Probe rheology approach}

Both NEMD and Green-Kubo approaches focus on the bulk properties of the material and extract its viscoelasticity from the stress signal calculated from the entire simulation cell. By contrast, the probe rheology approach, which is inspired by the experimental technique of microrheology [64,65], is local in nature and extracts the material viscoelasticity by tracking the dynamics of solid particles immersed therein [66-69]. There is a similar distinction between equilibrium and non-equilibrium techniques in probe rheology. In the equilibrium approach, commonly termed passive rheology, particle motions are driven by thermal fluctuations, whereas in the non-equilibrium approach - active rheology - an oscillatory external force is imposed on the probe particle whose average position is maintained by an additional harmonic trap potential.

For a solid particle moving in a viscoelastic medium, the equation of motion (EoM) can be written as a generalized Langevin equation (GLE) [26]. Dynamic moduli of the medium, $G^{\prime}$ and $G^{\prime \prime}$, are related to the friction coefficient in the drag force term via the generalized Stokes-Einstein relation (GSER) [70,71]. The resulting GLE in the frequency domain relates $G^{*}$ to the amplitude and phase lag of the particle position oscillation (in the active rheology approach) or to the Laplace transform of the particle MSD curve (in passive rheology) [66,69]. Khare and coworkers [66,69] demonstrated the importance of including the particle and medium inertia in the GSER without which the predicted $G^{*}$ would be incorrect and even unphysical at higher frequencies, especially in the case of $G^{\prime}$. Comparison was also made with the NEMD and GreenKubo approaches. It was found that $G^{\prime}$ and $G^{\prime \prime}$ calculated from the NEMD and GreenKubo approaches agree well with each other. Both the active and passive rheology results agree with these bulk approaches within a limited window of $\omega$.

A detailed analysis of the acceptable $\omega$ range was provided in ref. [69]. The lower bound of $\omega$ is determined by the distance over which the perturbation wave (caused by the particle motion) can penetrate into the surrounding medium. The penetration length increases with decreasing $\omega$ and for a sufficiently low $\omega$, the perturbation wave can travel across the polymer medium and reach the periodic image of the particle, causing hydrodynamic interaction between the periodic images, which is not accounted for in the current formalism. For entangled polymers, to capture $\omega$ down to $1 / \tau_{\mathrm{d}}\left(\tau_{\mathrm{d}}\right.$ is the disentanglement time - the longest relaxation time of entangled chains), the dimension of an adequate simulation cell (that prevents hydrodynamic interaction between images) is estimated to scale with $N^{3}$. The upper bound of $\omega$ is determined by the requirement that the probe particle size must be smaller than the perturbation 
wavelength to avoid ballistic particle motion (again beyond the range of applicability of the current formalism). Meanwhile, the particle size must also be large enough for the polymer medium to be considered a continuum $\left(>R_{\mathrm{g}}-\right.$ the polymer chain radius of gyration - for unentangled polymers and $\gtrsim O(1) d_{\mathrm{T}}$ - the confining-tube diameter for entangled polymers) [72,73]. All these constraints make it extremely challenging to obtain $G^{*}$ over a wide frequency spectrum. For the same system size of $\approx 2.87 \times 10^{6}$ beads, the probe rheology approach is reasonably accurate for about two dedaces of frequencies for short $N=20$ chains [66], whereas for $N=80$ chains, which are at most marginally entangled, the acceptable frequency range drops to about one decade [69]. In addition, the probe particle in MD simulation is modeled as a bundle of LJ beads and thus not strictly spherical. The choice of its effective diameter also affects the results and the most accurate choice seems to depend on the particle size itself [69].

These challenges, together with its more complex mathematical framework, seem to make the probe rheology approach a less appealing alternative to the bulk approaches discussed above. However, there is a unique advantage in its access to the spatial variations of materials properties, which is important for heterogeneous materials, such as polymer nanocomposites, micro-phase-separated block copolymers, and polymers near interfaces, especially in terms of establishing the relationship between the material microstructure and its local viscoelasticity.

\subsection{Chemically specific models}

The previous section reviewed major approaches for computing rheological properties of viscoelastic polymer melts from MD simulation, all using FENE bead-spring chains as a sandbox. The model was proposed as a minimalist representation of polymer chains containing generic features (at large scales) common to all polymers - chemical connectivity between monomers, entropic attraction between consecutive chain segments, and non-crossability between different segments - without specific correspondence to any real chemical structure. Comparison with experiments is possible if experimental data are available for mapping generic model parameters to specific polymer species. For example, one may obtain the molecular weight of the polymer segment represented by one bead by dividing the experimentally measured entanglement strand molecular weight $M_{\mathrm{e}, \exp }$ - from $G_{N}^{0}$ using eq. (16) - by the model entanglement strand length $N_{\mathrm{e}, \mathrm{KG}}$. Alternatively, the critical molecular weight/chain length for the onset of entangled behaviors (identified as a crossover point on the chain-length dependence of dynamical properties such as viscosity) $M_{\mathrm{c}, \exp } / N_{\mathrm{c}, \mathrm{KG}}$ can also be used [38]. Recent study by Takahashi et al. [28] further showed that using the information at $N_{\mathrm{c}}$ for parameterizing the KG model can faithfully capture the statics and dynamics of much longer chains. Nevertheless, the model cannot make any truly ab initio prediction: from chemical structure to rheological properties without experimental input (other than the experimental data required for force field parameterization, which generally come from common small-molecule benchmark compounds rather than the specific polymer of interest). MD prediction based on models constructed to represent specific chemical structures is reviewed here.

\subsubsection{Force-field based atomistic models}

Force fields for atomistic molecular models have been built for a wide range of chemical species by fitting the interaction energy profiles between atoms, calculated from quantum mechanics, to empirical potential energy formulae for bond, bond angle, torsion 
angle, etc. interactions [74-77]. The excluded-volume and van der Waals interactions are normally described with the LJ model which is often parameterized to match the results from molecular simulation with experimental structural and thermodynamic properties, such as density, heat of evaporation, and vapor-liquid equilibrium data. These models allow the direct mapping between a given chemical structure to a specific model, atom by atom. (Models that map every atom into one interaction site is called all-atom or explicit-atom force fields. Some force fields, such as TraPPE [77], group all $\mathrm{H}$ atoms to its host $\mathrm{C}$ atom and treat, e.g., each $\mathrm{CH}_{2}$ group as one interaction site. They are called united atom - UA - models.) Combining these atomistic models with the MD methods reviewed above can thus, in theory, directly predict rheological properties for any given polymer species, subject to the accuracy and availability of atomistic force fields. In practice, however, the range of applicability is severely limited by both the larger computational cost and the larger stress fluctuations in atomistic simulations.

2.2.1.1. Shear viscosity. Application of NEMD and Green-Kubo approaches for the shear viscosity of simple fluids predated most common general-purpose force fields used nowadays [78]. Increasing model complexity, brought by the explicit representation of each atom, leads to higher uncertainty in the results. Hess [79] compared a simple LJ fluid with short-range interactions with molecular water models (SPC and SPC/E [80]) and found that the EMD prediction of the shear viscosity, using the Einstein relation

$$
\eta_{0}=\frac{1}{2} \frac{V}{k_{\mathrm{B}} T} \lim _{t \rightarrow \infty} \frac{d}{d t}\left\langle\left(\int_{t_{0}}^{t_{0}+t} \sigma_{x y}\left(t^{\prime}\right) d t^{\prime}\right)^{2}\right\rangle_{t_{0}}
$$

(a reformulation of the Green-Kubo relation - eqs. (12) and (13)), contains larger uncertainty for molecular liquids. Prediction using atomistic models is sensitive to force-field settings. In particular, including long-range electrostatic interactions was found to be important.

As computer power grew over the decades, shear viscosity of larger and more complex molecules became within reach. Because of their simple molecular structure (which permits the usage of simple UA models) and wide availability of experiment data, alkanes have been extensively studied for viscosity prediction by MD. Daivis and Evans [81] extended the idea of eq. (17) and showed that by taking the traceless symmetric part of the stress tensor, six different SAFs, from the same EMD simulation, can be included in the average to improve precision. Mondello and Grest [82] extended this treatment to the Einstein relation and showed that its result matches that of the Green-Kubo relation. Comparison between a UA (TraPPE [77]) and allatom [83] force field for alkanes was reported by Payal et al. [84]. They found that the former estimates the shear viscosity within $20 \%$ to $30 \%$ of the experimental values whereas excellent agreement with experiments was found in the latter (all-atom) case. Shear viscosity of long-chain polymers has been reported recently with the NEMD approach $[85,86]$. In particular, Cho et al. [86] studied the shear flow of an entangled polyethylene $\mathrm{C}_{400} \mathrm{H}_{802}$ melt with an UA model and reported shear viscosity for over 4 decades of shear rates: from the Newtonian plateau $\left(\eta_{0}\right)$ to the shear-thinning power law region.

As far as shear viscosity is concerned, NEMD has the advantage over EMD approaches in terms of computational cost. Both Green-Kubo and Einstein relations 


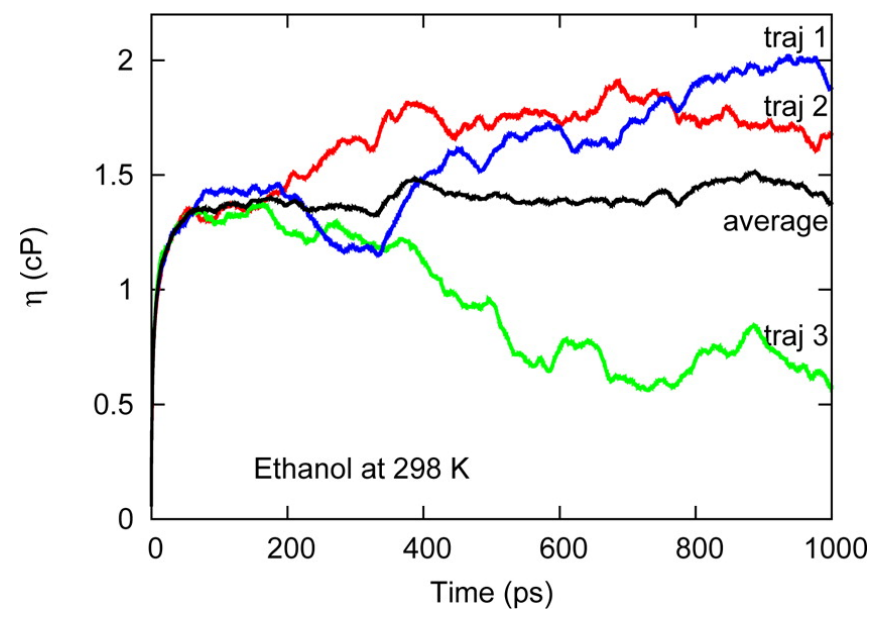

Figure 2. The truncated Green-Kubo integral for shear viscosity eq. (19) as a function of the integration upper limit $t^{\prime}$ for a molecular model of ethanol at $298 \mathrm{~K}$. Three independent trajectories are shown in different colors. The black line in the middle shows average over these trajectories. Large uncertainty is observed between independent trajectories after $O(100)$ ps. (Reprinted with permission from ref. [87]. Copyright (2015) American Chemical Society.)

require extended simulation runs to overcome the statistical uncertainty caused by stress fluctuation, which is only aggravated for molecular fluids. The advantage of equilibrium approaches is their ease of implementation. In addition, many other equilibrium properties of interest can be calculated in the same run [82].

Since the Green-Kubo approach for $\eta_{0}$ (eq. (13)) requires the integration of $G(t)$ to infinity, the finite-time truncation of the integral is an inevitable source of error. Hess [79] thus argued that the Einstein relation would be more reliable, although other studies found little noticeable difference between the two $[82,88]$. To evaluate the magnitude of this uncertainty, the finite time truncation of eq. (13)

$$
\eta_{0}\left(t^{\prime}\right)=\int_{0}^{t^{\prime}} G(t) d t
$$

can be calculated for different integration upper limit $t^{\prime}$ values (fig. 2). It was found that instead of converging to a steady value, the integral displays increasing uncertainty at higher $t^{\prime}[84,87,88]$. Indeed, the value obtained from a certain intermediate time range $(\approx 100$ ps in fig. 2$)$, where the curves have already flattened but statistics in the SAFs are still sufficient, is often closer to the average value than the long-time limit is. This indicates that the loss of accuracy caused by the truncation is much smaller than the statistical uncertainty in the SAF calculation at large time lags. This uncertainty is reduced after averaging over a number of independent MD trajectories.

A recent method proposed by Zhang et al. [87] takes advantage of the higher accuracy at the low- $t^{\prime}$ end and predicts the long-time plateau value of the integral (eq. (19)) by fitting its values to an empirical double-exponential approximation $[79,89]$

$$
\eta_{0}\left(t^{\prime}\right)=A \alpha \tau_{\mathrm{I}}\left(1-e^{-t^{\prime} / \tau_{\mathrm{I}}}\right)+A(1-\alpha) \tau_{\mathrm{II}}\left(1-e^{-t^{\prime} / \tau_{\mathrm{II}}}\right)
$$

where $A, \alpha, \tau_{\mathrm{I}}$, and $\tau_{\mathrm{II}}$ are model parameters. The running integral $\eta_{0}\left(t^{\prime}\right)$ is calculated with eq. (19) from multiple independent simulation runs (from different initial config- 
urations) and the diverging standard deviation between them is fitted to a power law function

$$
s_{\eta_{0}\left(t^{\prime}\right)}=A t^{\prime b}
$$

from which parameter $A$ in eq. (20) is obtained. The $t^{\prime}$ value at which the standard deviation $s_{\eta_{0}\left(t^{\prime}\right)}$ (as a measurement of uncertainty) exceeds $40 \%$ of the average is recorded as a cutoff. Independent $\eta_{0}\left(t^{\prime}\right)$ profiles before the cutoff are averaged and used to fit eq. (20) (data points at different $t^{\prime}$ are weighted by $1 / t^{\prime b}$ ) to obtain the remaining model parameters. Shear viscosity is then predicted from the $t \rightarrow \infty$ limit of eq. (20). The method was applied to simple liquids with viscosity ranging from $O(1) \mathrm{cp}$ to $O(100) \mathrm{cp}$ and its predictions agreed well with experiments [87]. Extension of this approach to polymer chain molecules, especially in the entangled regime, will likely require a different convergence profile (than eq. (20)) because of their different relaxation characteristics.

2.2.1.2. Linear viscoelasticity. The full spectrum of linear viscoelasticity, represented by $G^{*}(\omega)$ or $G(t)$, is intrinsically more demanding to compute than shear viscosity. (For $\eta_{0}$ using eq. (13), positive and negative noises in the $G(t)$ signal cancel over a long-time integration, whereas for $G^{*}(\omega)$ - eqs. (14) and (15) - accuracy at all frequencies is required.) For long-chain polymers, calculation of these quantities at the all-atom level, using either equilibrium or non-equilibrium approaches, is still computationally prohibitive even today. Earlier studies mostly simulated short unentangled chains using UA models and focused on the dynamics in the short-time (high-frequency) limit. Rheological models were used to extend the prediction to longer times (see section 3). Harmandaris et al. [90] used the Green-Kubo approach to compute $G(t)$ only for short-chain $\left(\mathrm{C}_{24}\right)$ UA polyethylene and for very short time lags (up to $O(10)$ ps). The Rouse model was invoked to extend the prediction to longer times (see section 3.1). Byutner \& Smith [91] used the same approach for a UA polybutadiene $\mathrm{C}_{114}$ to compute $G^{\prime}$ and $G^{\prime \prime}$ for the $\omega>O\left(10^{-5}\right) \mathrm{ps}^{-1}$ region (fig. 6), within the glassy regime and part of the Rouse regime, and the results agreed well with experimental measurements of much longer (entangled) chains.

In recent years, atomistic simulation of viscoelastic behaviors of asphalt has received much attention. Asphalt is a complex mixture of up to millions of organic compounds of three major types - asphaltenes, saturates, and resins (aphthene and polar aromatics) $[92,93]$. In molecular simulation, asphalt is typically modeled by a small group of representative compounds: e.g., the recent model of Li \& Greenfield [94] contains 12 compounds with molecular weight up to $\sim 900$ Da. Khabaz \& Khare [95] used NEMD to calculate the shear viscosity, dynamic moduli, and creep compliance of that model. It was found that the time-temperature superposition (TTS) principle [96] was applicable to the system with consistent shift factor $\alpha_{T}$ values obtained from different rheological properties. Applying TTS allowed the construction of $G^{\prime}$ and $G^{\prime \prime}$ master curves spanning over 6 decades of frequencies while the original NEMD results only covered slightly over one decade at each temperature level. For the Green-Kubo approach, Masoori \& Greenfield [97] observed enormous stress fluctuations in the atomistic MD of the asphalt model: a simple 100-step (0.1 ps) moving average is not adequate to yield meaningful results. To counter the spectral leakage effect, which occurs during the Fourier transform of $G(t)$ (to obtain $G^{*}(\omega)$; see eqs. (14) and (15)) over a finite integration window, an exponentially decaying window function was applied by the 


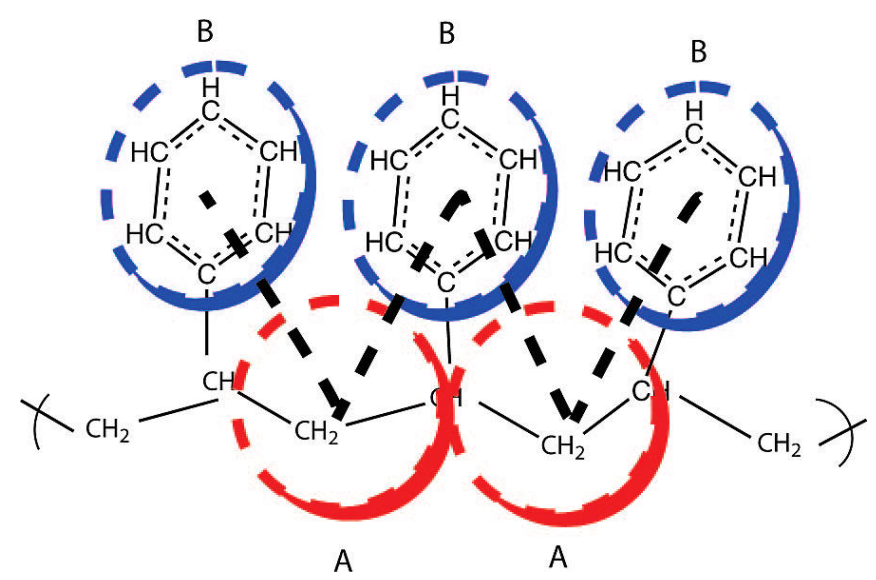

Figure 3. A low-level coarse-graining (CG) mapping scheme for polystyrene. Two types of CG beads (superatoms) are used: type A (red) for the carbon backbone segment and type B (blue) for the benzene ring; thick dashed lines represent the CG bonds between bead types A and B. (Reprinted with permission from ref. [98]. Copyright (2009) American Chemical Society.)

authors to force the vanishing of $G(t)$ within the window. (It was later compensated for in the frequency domain $G^{*}(\omega)$ - adapting the method to entangled polymers with drastically different relaxation profiles may require a different approach). This measure was used together with window average in the time domain $(G(t))$ and the removal of unphysically low stress values in the frequency domain $\left(G^{*}(\omega)\right)$ to obtain the best noise reduction in the dynamic moduli and phase angle $\delta$.

\subsubsection{Systematic coarse-graining}

Atomistic molecular models retain all degrees of freedom (DoFs) of the physical system and are thus computationally most demanding. To fully capture the whole spectrum of polymer dynamics (which, as discussed above, spans a wide range of scales), a hierarchical modeling approach becomes an inevitable choice [99], which uses the more detailed models to capture the small scales and coarser models for larger scales. The whole spectrum of dynamics will be recovered if pieces of information from different levels are consistently stitched together.

A CG molecular model reduces the computational cost by projecting the molecular structure to much fewer DoFs, in which each CG bead (interaction site in the model) represents multiple atoms - from a functional group or fragment in a repeating unit for low-level models (fig. 3) to a large polymer chain segment in high-level ones. The $\mathrm{CG}$ potential (or force field) is parameterized to reflect the specific chemical structure it is representing, which can take both bottom-up and top-down strategies (and sometimes a combination of the two). The latter approach is much like the parameterization of non-bonded interaction terms in classical atomistic force fields (see, e.g., ref. [76]), which determines the interaction parameters between CG beads by matching the model prediction with experimental thermodynamic data. One well-known such example is the CG MARTINI force field [100]. Meanwhile, the bottom-up approach constructs CG potentials by systematically pipelining information from detailed models at the atomistic level, which thus allows the $a b$ initio prediction of materials properties without additional experimental input. 
2.2.2.1. Construction of the $C G$ potentials. Major strategies for the systematic bottom-up CG process have been extensively reviewed in the literature [101-106]. For bonded interactions between chemically-connected CG beads, Boltzmann inversion (BI) is among the most widely-used approaches: e.g., the bond stretching potential $U_{\text {bond }}^{\mathrm{CG}}\left(r_{\text {bond }}^{\mathrm{CG}}\right)$ between two connected CG beads is obtained by inverting the Boltzmann distribution function of the $\mathrm{CG}$ bond length $r_{\text {bond }}^{\mathrm{CG}}$

$$
U_{\text {bond }}^{\mathrm{CG}}\left(r_{\text {bond }}^{\mathrm{CG}}\right)=-k_{\mathrm{B}} T \ln P\left(r_{\text {bond }}^{\mathrm{CG}}\right)
$$

where $r_{\text {bond }}^{\mathrm{CG}}$ is measured by the distance between the CG bead centers in an atomistic benchmark simulation (usually using shorter chains and a smaller simulation cell) and $P\left(r_{\text {bond }}^{\mathrm{CG}}\right)$ is its distribution from the benchmark run. Bond angle and torsion angle (dihedral) interactions (if present in the CG model) are similarly obtained. Non-bonded interactions are often optimized to match the structural or thermodynamic properties of the atomistic benchmark simulation, using methods such as iterative Boltzmann inversion (IBI) or inverse Monte Carlo (IMC). Both IBI and IMC iteratively update the pairwise interaction $U_{\text {pair }}^{\mathrm{CG}}(r)$ until the chosen property converges to the atomistic result. Take the IBI approach for matching the bead radial distribution function (RDF) $g(r)$ for example. During each iteraction,

$$
U_{\mathrm{pair}, i+1}^{\mathrm{CG}}(r)=U_{\mathrm{pair}, i}^{\mathrm{CG}}(r)+k_{\mathrm{B}} T \ln \left(\frac{g_{i}^{\mathrm{CG}}(r)}{g_{\mathrm{ref}}^{\mathrm{CG}}(r)}\right)
$$

where $U_{\text {pair }, i}^{\mathrm{CG}}(r)$ and $U_{\text {pair }, i+1}^{\mathrm{CG}}(r)$ are the pairwise potential at the $i$ - and $(i+1)$-th iteration step, respectively, $g_{i}^{\mathrm{CG}}(r)$ is the RDF obtained in the $i$-th step simulation using $U_{\mathrm{pair}, i}^{\mathrm{CG}}(r)$, and $g_{\mathrm{ref}}^{\mathrm{CG}}(r)$ is the reference RDF (from the atomistic benchmark). The method can be modified to add additional target properties, such as thermodynamic properties, for their simultaneous optimization [107]. In addition to these potentialbased approaches, the CG force field can also be optimized to match forces on the CG beads with those of the atomistic benchmark $[108,109]$.

Systematic CG approaches face challenges such as the limited transferability of CG potential parameters between thermodynamic states: parameters obtained at one state may not be accurate for another. Also, different properties are not always consistently predicted.

2.2.2.2. Mapping of the dynamics. For predicting dynamical properties such as diffusivity, heat conductivity, and, in the context of this review, rheological properties, an important factor not to be overlooked is the artificial acceleration of the dynamics at the CG level $[110,111]$. The reduction of DoFs in the CG process reduces the ruggedness of the potential energy surface and causes a sharp decrease of the intermolecular friction (compared with the original atomistic system), which leads to the unphysical speed-up of molecular motions. Additional measures are required to counter this effect.

2.2.2.2.1. Time rescaling. At lower levels of $\mathrm{CG}$, a simple workaround is to apply a time rescaling factor obtained heuristically by matching the diffusivities from the two model levels [110]. Although there is no guarantee that different dynamical processes in the system scale by the same factor, the agreement between the CG and atomistic MSD curves does extend beyond the diffusive regime (where the fitting is performed) to much of the short-time subdiffusive regime. Harmandaris \& Kremer [112] constructed 
a CG model for polystyrene that maps each repeating unit to two CG beads with BI (fig. 3). It was found that the same scaling factor is able to match both the MSD and end-to-end vector autocorrelation function between the CG and atomistic models: indicating a consistent level of CG-induced acceleration between chain diffusion and conformational relaxation dynamics [98]. The time scaling factor is a function of chain length but converges to a plateau at the long-chain limit, which allows its estimation using a moderate chain length at the atomistic level for the CG simulation of long chains [113]. For structurally or dynamically heterogeneous systems - such as mixtures, composite materials, or polymers near $T_{\mathrm{g}}$ - or for non-equilibrium dynamics, a singlevalue scalar factor may no longer be applicable [113]. In an ethylbenzene-polystyrene binary mixture, it was found that the scaling factor for the polymer is over one order of magnitude higher than that of the solvent [110].

2.2.2.2.2. Friction coefficient. At higher levels of $\mathrm{CG}$ (in which many repeating units map to each CG bead), explicitly introducing the friction drag and - by the fluctuationdissipation theorem [26] - random forces into the EoM becomes necessary. These forces can be either pairwise, as in dissipative particle dynamics (DPD) [114], or between the CG beads and a background medium, as in Brownian dynamics (BD) [115]. Maurel et al. [116] built a CG DPD model for cis-1,4-polybutadiene using a UA model as the benchmark. Each DPD bead maps to 5 repeating units (20 backbone carbons) and the CG potentials (conservative force in DPD) were generated through the IBI approach (for both bonded and non-bonded interactions). A pressure correction term to the non-bonded interaction (proposed by Reigh et al. [117]) was found necessary to reproduce the correct thermodynamic state. The friction coefficient was left as a free parameter and chosen somewhat arbitrarily: the dynamics of the model thus does not map directly to the experimental system. The relaxation modulus $G(t)$ was computed from the DPD trajectory with the Green-Kubo approach using the Ramírez et al. [62] multiple-tau correlator algorithm for the SAF. The longest chain studied was $\mathrm{C}_{800}\left(\approx 4 N_{\mathrm{e}} ; N_{\mathrm{e}}\right.$ was estimated from the primitive path analysis [48] - see below in section 3.2.1), for which no clear stress plateau was observed. This is consistent with the earlier discussion that a clear-cut stress plateau would not occur for $N<O(10) N_{\mathrm{e}}$ (see section 2.1.2).

Several efforts have been made to systematically extract the lost friction from the DoFs eliminated in the CG process. Using a model binary LJ fluid mixture as the benchmark, Shell [118] showed that his relative-entropy formalism (that the relative entropy between the CG and reference systems is the fundamental quantity to minimize in a rigorous hierarchical modeling framework [119]) consistently retains the thermodynamic and dynamic properties of the liquid. For molecular liquids, friction force parameterization has been developed in the framework of the force-matching CG strategy $[108,109]$. These methods, however, were so far mostly demonstrated in nonpolymeric simple liquid model systems. Izvekov \& Voth [120] used the force-velocity and velocity-velocity time correlation functions in the atomistic benchmark simulation to obtain the friction coefficients in the GLE for the CG system. Diffusion coefficient of methanol computed from the CG BD accurately reflected the all-atom result. In another approach developed by Markutsya \& Lamm [121], which is more efficient for complex liquids and mixtures containing many different CG bead types, the friction coefficient in the Markovian Langevin equation (which, compared with the GLE, neglects the memory effect in the friction coefficient by assuming its faster decay than the time scale of bead motion) is extracted from the fluctuations of interaction forces 


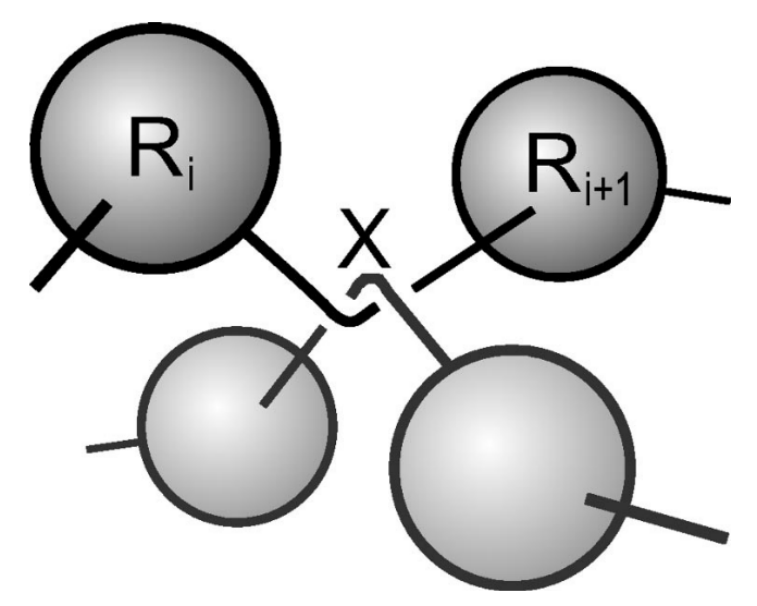

Figure 4. Schematic of an entanglement (bond-crossing) event detected by the TWENTANGLEMENT algorithm [122]. A crossing point $\vec{X}$ is recorded, which is allowed to move along both bonds. Calculation of the bond energies of the intersecting bonds considers the stretched/kinked contour length, until disentanglement is detected. (Reprinted from ref. [124], with the permission of AIP Publishing.)

between CG beads. (Force fluctuations are recorded in the same atomistic benchmark simulation used to compute the average forces for the CG potential). The method was tested on the pure TIP3P water model [80] and a glucose in TIP3P water solution. Compared with conventional CG MD (i.e., without friction and random forces), which overestimates the molecular diffusivity by one order of magnitude, the $\mathrm{BD}$ simulation with friction coefficients derived from this approach agrees excellently with the atomistic benchmark.

2.2.2.2.3. Non-crossability - preserving the chain topology. For polymers, Padding \& Briels $[22,122-124]$ have developed a hierarchical modeling framework for the prediction of their rheology and dynamical behaviors. It builds CG mesoscopic models for long entangled polymers following an ab initio paradigm - completely bottom-up using the atomistic simulation as the only source of information.

A new element introduced in the model, compared with other systematic CG approaches, is the explicit treatment of the entanglements between CG bonds. In their framework, the term "entanglement" is taken at its face value: topological crossing (more accurately, attempted crossing) of bonds that results in their intertwinement. Explicit inclusion of entanglements in the EoM is necessary to enforce the non-crossability between polymer chains at high levels of $\mathrm{CG}$, which they referred to as CG stochastic dynamics (CGSD), in comparison to CGMD at lower levels of CG [22]. CG potentials soften with the increasing CG level. For CGMD (e.g., the example of fig. 3), in which each bead acts as a "superatom" grouping a limited number of real atoms (typically less than 5 backbone carbons plus other atoms attached thereon), their interactions are still hard enough to prevent unphysical chain crossing. For dynamic mapping of these models, a time rescaling approach would suffice in most homogeneous systems. For CGSD, each bead represents a "blob" containing a large chain segment and their potentials become too soft to preserve the topological non-crossablilty between chains. Models at this level typically require the explicit consideration of friction, and thus random, forces in the EoM, thus the word "stochastic" in its the name.

A so-called "TWENTANGLEMENT" algorithm was proposed for bond crossing 
detection [122] (fig. 4). Once detected, the algorithm adds a crossing point that alters the dynamics of the four beads involved in the two crossing bonds. The crossing point is allowed to glide along the bonds. The potential energies of the entangled bonds are calculated using the now kinked bond contour length

$$
L_{i, i+1}=\left|\vec{R}_{i}-\vec{X}\right|+\left|\vec{X}-\vec{R}_{i+1}\right|
$$

where $L_{i, i+1}$ is the bond length between the $i$ - and $(i+1)$-th atoms, with position vectors $\vec{R}_{i}$ and $\vec{R}_{i+1}$, and $\vec{X}$ is the position vector of the crossing point. This energy contribution prevents the phantom crossing of the bonds. The normal bond settings will be restored once disentanglement is detected. (A conceptually similar method for preventing bond-crossing in $\mathrm{BD}$ simulations was reported, at around the same time, by Kumar \& Larson [125]. Compared with the TWENTANGLEMENT algorithm, it does not directly account for the changing potential after entanglement. Instead, it adds a repulsive potential between bonds that prevents entanglement from ever happening.)

CG potentials were built for polyethylene through BI, in which each bead maps to 20 backbone carbon atoms. (For the same 20 carbon per bead mapping, ref. [116], discussed above, did not detect chain crossing - using the method of Goujon et al. [126] - and thus found no need for additional topology preservation measures. This discrepancy may be attributed to the difference in backbone rigidity between cis-1,4polybutadiene and polyethylene.) Friction coefficients, used in the CG BD simulation, were systematically extracted from the random force autocorrelation function using a probe-bead method later introduced into the framework [123]. In this method, a group of atoms in the atomistic benchmark represented by a CG bead (one "blob") are fixed by a constraining force, which balances the random forces from (1) interactions with all other atoms and (2) coupling with the thermostat (heat bath). The two contributions are separated based on a cutoff frequency determined from the CG potential of mean force profiles. Contribution (1) goes into the friction coefficient parameterization.

Other than the atomistic force field, the resulting model does not contain any adjustable parameter that is not determined a priori. It accurately predicts (compared with experimental values) the shear viscosity and diffusivity of polyethylene for chain lengths up to $\mathrm{C}_{1000}$, including both unentangled and entangled cases. The $G(t)$ was computed with the Green-Kubo relation and a stress plateau seems to appear at $\mathrm{C}_{400}$ (in the midst of strong noises). The entanglement strand length and plateau modulus were both accurately predicted. Application to the non-equilibrium simulation of both steady and transient shear flows was also reported [124] and the results agreed with available experimental data.

\section{Integration with rheological models}

The biggest challenges in the prediction of polymer rheology by molecular simulation, including the large uncertainty caused by the intense stress fluctuations and the exploding time scales of long-chain dynamics, can be circumvented when molecular simulation joins forces with appropriate rheological models for an integrated multiscale predictive framework. In these approaches, molecular simulation is used to provide materials-specific information to complement generic rheological models. Model-based predictions are always smooth (free from noise) and the time scales they can reach are not as limited by computational resources as they are by the model's range of applicability. Of course, prediction reliability is necessarily limited by the accuracy 
of the rheological models themselves. For example, both the Rouse and tube models take a mean-field approach that describes the relaxation of a probe chain submerged in a continuous background formed by all surrounding chains. Correlation between the relaxation dynamics of different chains cannot be straightforwardly accounted for. Such effects are more clearly observed in bidisperse mixtures containing chains of two different lengths, where the cross-species correlation between the segmental orientations of the two chain types was found to decay over the relaxation time of the longer chains [127]. Continued development and improvement of rheological models are the key to the prediction accuracy of all approaches discussed in this section.

\subsection{Unentangled dynamics: the Rouse model}

The Rouse model is widely used for the dynamics of unentangled polymer melts. It describes the dynamics of a probe chain, modeled as a string of beads connected by Gaussian (compared with FENE in the KG model) springs, submerged in a viscous medium representing the collective effects of all surrounding chains $[17,46]$. In addition to its highly-simplified representation of the molecular structure, the model also mostly neglects non-bonded monomer-monomer interactions including the excluded-volume and hydrodynamic interactions. Interactions between the non-bonded monomers in the probe chain are completely neglected, whereas its interactions with surrounding chains are described through its Brownian motion in the medium. Bead coordinates of the model chain are projected to a set of normal modes and each mode relaxes exponentially with its own relaxation time. The stress relaxation modulus $G(t)$ can be correspondingly decomposed into a spectrum of relaxation modes

$$
G(t)=\frac{\nu k_{\mathrm{B}} T}{N} \sum_{p=1}^{N} \exp \left(-\frac{2 t}{\tau_{p}}\right)
$$

where $\nu$ is the number density of the beads, $p$ is the Rouse mode index, and $\tau_{p}=\tau_{1} / p^{2}$ is the relaxation time of the $p$-th mode $\left(\tau_{1}\right.$ is the relaxation time of the slowest mode which is the Rouse time $\tau_{\mathrm{R}}$.).

Molecular simulation data can be used to parameterize the Rouse model for rheological prediction. For the CG simulation of bead-spring chain models, time-dependent Rouse modes can be directly calculated knowing the coordinates of each bead. Vladkov \& Barrat [44] explored this Rouse mode analysis (RMA) as an alternative approach for computing linear viscoelasticity (of the KG FENE chain model) that avoids the strong noise and uncertainties found in direct MD results. The obtained Rouse modes were used to calculate the equilibrium stress, which, through the Green-Kubo relation, produces $G(t)$ and thus $\eta_{0}, G^{\prime}$, and $G^{\prime \prime}$. For two different chain lengths ( $N=10$ and 20 ), the RMA prediction consistently underestimated (compared with the Green-Kubo result from direct $\mathrm{MD}$ ) $\eta_{0}$ by the same error magnitude. It was thus inferred that RMA preserves the stress contribution from chain connectivity (the "polymer" contribution) but leaves out that of monomer-monomer interactions (the "non-polymer" contribution). The latter is associated with individual beads and thus independent of chain length. The missing contribution is from time scales below the relaxation time of the $(N-1)$-th Rouse mode $\tau_{N-1}$ and can be effectively compensated by adding

$$
\int_{0}^{\tau_{N-1}} \frac{V}{k_{\mathrm{B}} T}\left\langle\sigma_{x y}(t) \sigma_{x y}(0)\right\rangle d t
$$


(cf. eq. (12)) to the RMA prediction, where $\sigma_{x y}$ is from direct EMD. For the dynamic moduli, the RMA can closely reproduce $G^{\prime}$ compared with NEMD results but it underestimates $G^{\prime \prime}$ especially at the high frequency end. A correction was similarly introduced in which the short-time part of the $G(t)$ profile from direct EMD was fitted to an empirical expression and the correction terms to $G^{\prime}$ and $G^{\prime \prime}$ were obtained from the resulting $G(t)$ expression using eq. (15).

For atomistic polyethylene $\left(\mathrm{C}_{20}\right.$ to $\mathrm{C}_{150}$; UA model $)$, Harmandaris et al. [128] calculated the autocorrelation functions of the Rouse modes from MD and, by fitting these autocorrelations to an exponential decay function, extracted their relaxation times $\tau_{p}$. Equation (25) was then invoked to calculate $G(t)$. For two chain lengths $\left(\mathrm{C}_{46}\right.$ and $\mathrm{C}_{90}$ ) where experimental data were found, the Rouse prediction underestimated $\eta_{0}$ by $0.6 \mathrm{cp}$ to $1.0 \mathrm{cp}$. In a later study by the same authors [90], relaxation dynamics of the same polyethylene model $\left(\mathrm{C}_{24}\right.$ and $\left.\mathrm{C}_{78}\right)$ was investigated with an NEMD approach. Pre-strained polymer configurations subject to a steady-state extensional deformation was generated with an end-bridging Monte Carlo (EBMC) algorithm [5] and their relaxation to equilibrium was simulated with MD. RMA was used to extract the relaxation time spectrum $\tau_{p}$ from the stress relaxation of the pre-strained conformation - which takes only half of the time compared with equilibrium MD - and $G(t)$ is again obtained from eq. (25). Direct computation of $G(t)$ from EMD was also performed for the $\mathrm{C}_{24}$ case in the short-time-lag $(t \lesssim O(10 \mathrm{ps}))$ limit. The RMA profile appeared to extend continuously from the EMD one within statistical uncertainty. However, statistical noises at the end of the $G^{\mathrm{EMD}}(t)$ profile $(t \approx 40 \mathrm{ps})$ span over a wide range of $O\left(10^{6}\right) \sim O\left(10^{8}\right) \mathrm{Pa}$ and the $G^{\mathrm{RMA}}(t)$ magnitude at the same time lag was closer to the lower bound of the fluctuations than to the mean. This observation is still consistent with the conclusion of ref. [44] that RMA without correction underestimates the relaxation stress. Padding \& Briels [123] used a more detailed expression for $G(t)$ (than eq. (25)). It explicitly incorporates the normal coordinates of the Rouse modes into the prefactors of the relaxation modes. Each mode decays with time following a stretched exponential function. The normal coordinates and relaxation times of Rouse modes were extracted from their CGSD model of polyethylene. The prediction was extended to the entangled regime $\left(t>\tau_{\mathrm{e}}\right)$ by invoking the original tube model [46], which was fused with the Rouse expression by requiring the continuity of $G(t)$ at $t=\tau_{\mathrm{e}}$. The plateau modulus $G_{N}^{0}$ predicted from the combined models is close to (but lower than) the results of direct $\mathrm{BD}$ (calculated with the Green-Kubo relation).

Existing attempts of invoking the Rouse model to assist with rheological prediction seem to consistently underestimate the relaxation modulus, likely caused by its simplifications. Although remedies have been proposed (in ref. [44]) to correct for the lost information at the sub-Rouse small scales $\left(t<\tau_{N-1}\right)$, it likely does not account for all its errors. Notably, Masubuchi et al. [129] recently reported that both the FENE bead-spring model and all-atom models deviate from the Rouse dynamics starting from the second mode $(p=2)$.

\subsection{Entangled dynamics: tube and slip-link/-spring models}

The dynamics of polymer chains undergoes a distinct transition upon a critical chain length marked by, among many others, a sharp turn in the $\eta_{0}$ vs. $N$ relationship (from $\eta_{0} \propto N$ to $\eta_{0} \propto N^{3.4}$ ) and the emergence of a stress relaxation plateau in the $G(t)$ profile. This is caused by the topological constraints imposed by the non-crossability of polymer chains, commonly described as "entanglements", which drastically slow 

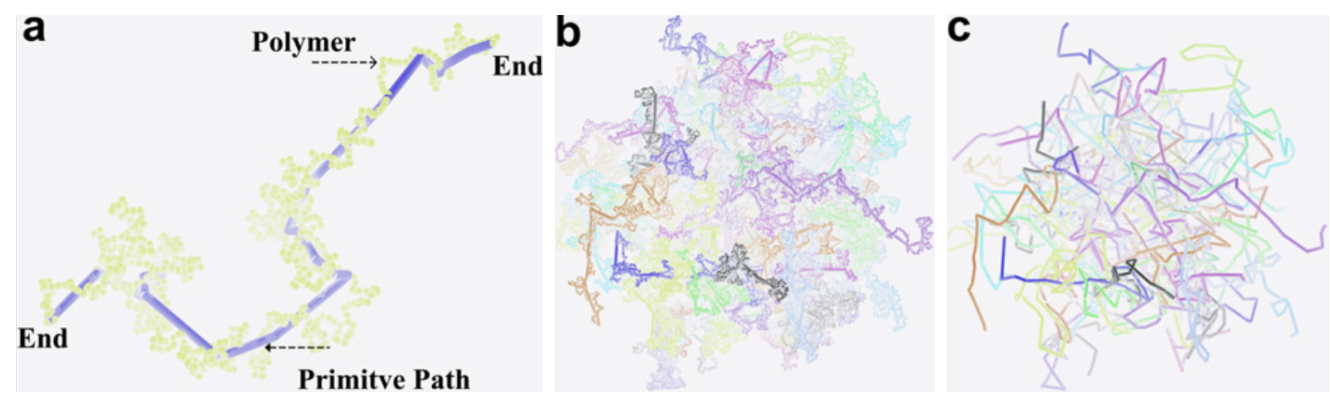

Figure 5. Primitive paths (PPs) extracted from an all-atom polyisoprene model using the Z1 code [132]: (a) the original chain (light/green) and PP (dark/blue) of an individual polymer molecule; (b) all PPs in a cell shown with the original chains; and (c) PPs only. Different colors are for different chains. Kinks in the PPs represent points of entanglement. (Reprinted from ref. [133], with permission from Elsevier.)

down the chain relaxation dynamics. Capturing the dynamics of polymer chain in this regime using brute-force MD, even with state-of-the-art CG techniques, would be an endless chase as the longest relaxation time $\tau_{\mathrm{d}} \propto N^{3.4}$. Rheological models for entangled polymers become a necessary bridge that connects short-time molecular simulation to long-time dynamics.

\subsubsection{Extending molecular simulation with tube models}

Since its inception circa five decades ago $[130,131]$, the tube theory has remained the most popular description of entangled polymer dynamics as it offers a reasonable balance between conceptual simplicity and predictive capability. It takes a mean-field approach and treats the topological constraints imposed by all other chains surrounding a probe chain as a confining potential, which restricts its lateral motions and forces its relaxation to the longitudinal direction. The potential contour has the shape of a curvilinear tube and its axis, where the confining potential reaches its minimum in each cross-sectional plane, is termed the "primitive path (PP)" (fig. 5 \& fig. 7(a)). The one-dimensional diffusion of the chain along the tube can be solved to give an $\eta_{0} \propto \tau_{\mathrm{d}} \propto N^{3}$ scaling which falls short of the 3.4 exponent found experimentally [46]. Inaccuracy of the original tube model led to the introduction of numerous modifications and improvements over the years $[17,18]$. Two most notable additions to the model are (1) contour length fluctuations (CFL; segmental displacements at both ends of the tube which lead to the fluctuations in the tube contour length) and (2) constraint release (CR; disentanglement of surrounding chains causes changes in the tube boundary itself).

3.2.1.1. Primitive path analysis. The primitive path (fig. 5), according to the original concept of Edwards [134], is obtained when the probe chain is contracted, with its both ends tethered, in a forest of line obstacles (representing the topological constraints imposed by all its surrounding chains) while preserving all non-crossablilty constraints with those obstacles. Several computer algorithms have been developed to extract the PPs from the polymer configurations obtained from molecular simulation.

The first such algorithm, developed by Everaers and coworkers $[48,135]$, takes an energy annealing approach. It performs energy minimization with all chain ends fixed in space and all intramolecular non-bonded interactions switched off. This allows the bond forces to contract the chains to their minimal contour lengths, subject to the topological constraints imposed by other chains since intermolecular interactions are 
still kept on. The resulting PPs, represented by the contracted bead-spring chains, are mapped to a freely-jointed chain (FJC) model to extract the entanglement statistics. Consider each PP an $\left(N / N_{\mathrm{e}}\right)$-step random walk with step length $d_{\mathrm{T}}$ (tube diameter measured by the end-to-end distance of entanglement strands):

$$
\left.\begin{array}{l}
\left\langle R^{2}\right\rangle=\left(\frac{N}{N_{\mathrm{e}}}\right) d_{\mathrm{T}}^{2} \\
\left\langle L_{\mathrm{pp}}\right\rangle=\left(\frac{N}{N_{\mathrm{e}}}\right) d_{\mathrm{T}}
\end{array}\right\} \Rightarrow\left\{\begin{array}{l}
d_{\mathrm{T}}=\frac{\left\langle R^{2}\right\rangle}{\left\langle L_{\mathrm{pp}}\right\rangle} \\
N_{\mathrm{e}}=N \frac{\left\langle R^{2}\right\rangle}{\left\langle L_{\mathrm{pp}}\right\rangle^{2}}
\end{array}\right.
$$

where $\left\langle L_{\mathrm{pp}}\right\rangle$ and $\left\langle R^{2}\right\rangle$ are the ensemble-average contour length and square end-to-end distance of the PPs (the latter is the same as the square end-to-end distance of the original chain, whereas $\left\langle L_{\mathrm{pp}}\right\rangle<L$ - the contour length of the original chain).

Two other algorithms were developed shortly after: the Z1 code by Kröger [132] and the CReTA algorithm by Tzoumanekas et al. [136]. These two are very similar in their approaches and both are based on geometric minimization, which simultaneously minimizes the contour lengths of all chains with their ends held fixed and all topological constraints preserved. Geometric minimization is several orders of magnitude faster than energy annealing [137] and can be used to extract the full distribution of entanglement strands. The PPs obtained are piecewise linear lines and the kinks between line segments represent points of entanglement. The entanglement points can be mapped to the original chains, allowing the direct identification of individual entanglement strands thereon. (For the energy annealing approach, whose resulting PPs are not line representations but bead-spring chains, Shanbhag \& Larson [138] have proposed a method to identify the entanglement points based on the relative positioning of neighboring beads.)

The individual entanglement strand length measured this way $\left(N_{\mathrm{e}}^{\text {indv }}\right)$ is within the same order of magnitude of but differs substantially from the value estimated with eq. (27). This indicates that PPs are not strictly FJCs and the orientations of consecutive strands/segments are correlated. Estimation from eq. (27) should thus be interpreted as the Kuhn length of the PPs $\left(N_{\mathrm{e}}^{\mathrm{Kuhn}}\right)$. Using a theoretical argument based on the phantom network model for rubber elasticity, Everaers [139] showed that the two measurements are related by

$$
N_{\mathrm{e}}^{\mathrm{Kuhn}}=\frac{N_{\mathrm{e}}^{\mathrm{indv}}}{\left(1-\frac{2}{f}\right)}
$$

where $f$ is the functionality of the elastic junctions - number of strands connected to each junction point. For entanglements between two chain segments, $f=4$, leading to $N_{\mathrm{e}}^{\mathrm{Kuhn}}=2 N_{\mathrm{e}}^{\mathrm{indv}}$ (which is consistent with multiple simulation findings using both CG and atomistic models). It was further argued that $N_{\mathrm{e}}^{\mathrm{Kuhn}}$ is the correct value to use for calculating the plateau modulus with eq. (16). Establishing such a relation between $N_{\mathrm{e}}$ values from different measurements is important not only for the interpretation of rheological models but also for their application in the $a b$ initio prediction of rheological properties. For the latter, both the tube (section 3.2.1) and slip-link/-spring (section 3.2.2) models are being used as a bridge between chemically-specific molecular models and the long-time relaxation dynamics of entangled polymers. Accurate determination of $N_{\mathrm{e}}$ is essential for prediction reliability. Indeed, $N_{\mathrm{e}}$ is still chosen with some level of arbitrariness in many studies.

Comparison between different PP analysis methods have been extensively discussed 


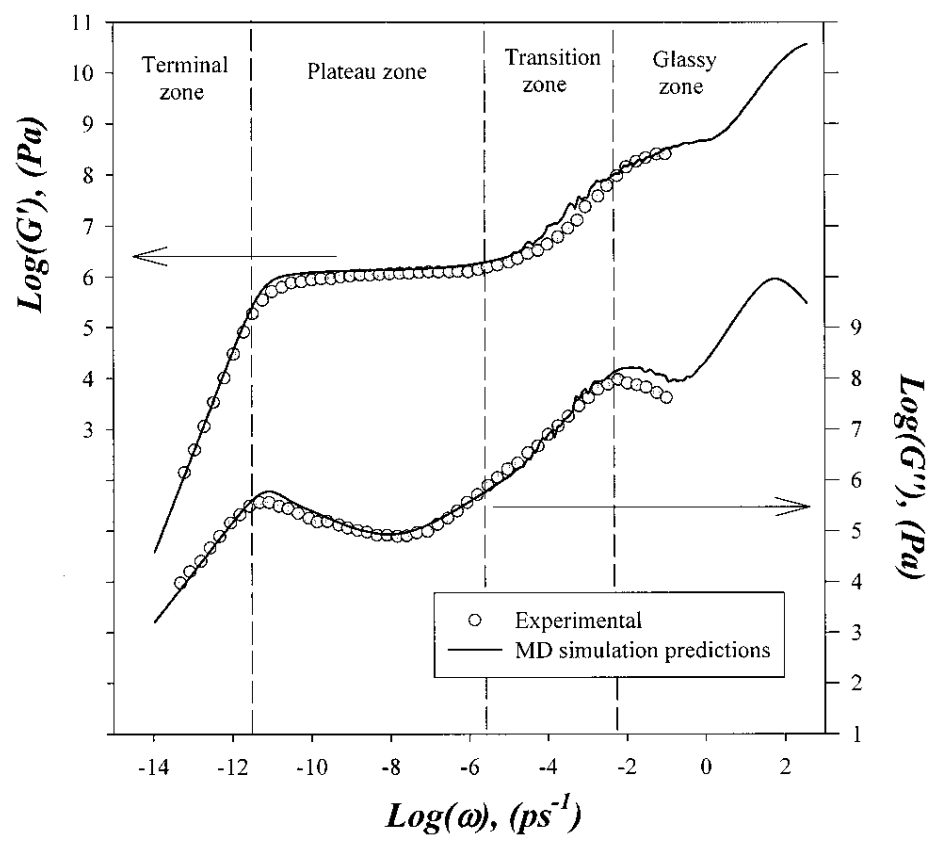

Figure 6. Dynamic moduli $G^{\prime}$ and $G^{\prime \prime}$ of polybutadiene (PBD; molecular weight $\left.M_{\mathrm{w}}=1.3 \times 10^{5} \mathrm{Da} ; 298 \mathrm{~K}\right)$ : comparison between experiments (circles) and MD simulation/tube model predictions (lines; using the Byutner $\&$ Smith procedure [91]). The MD simulation used short unentangled PBD chains $\left(\mathrm{C}_{114} ; M_{\mathrm{w}} \approx 1540 \mathrm{Da}\right)$ and covers the glassy regime (high $\omega$ ) in the plot; the Milner \& McLeish [141] tube model was used to extend the prediction to lower frequencies (parameterized by the MD simulation - see section 3.2.1). (Reprinted with permission from ref. [91]. Copyright (2002) American Chemical Society.)

in the literature: see discussion in ref. [136], a comparative study by Shanbhag \& Kröger [137], and the review article by Tzoumanekas \& Theodorou [140]. Application of PP analysis in nonlinear shear flow was also reported [85], offering important insight for the development of rheological models for strongly non-equilibrium flows.

3.2.1.2. Multiscale predictive frameworks built on tube models. Byutner \& Smith [142] proposed a comprehensive framework that fuses together the DavidsonCole model for glassy dynamics [143] (short time/high frequency), Rouse model for the relaxation of entanglement strands (intermediate time/frequency), tube models for reptation (long time/low frequency) to predict the whole spectrum of linear viscoelastic behaviors of entangled polymers. Various theoretical and, sometimes, empirical relations were invoked to minimize the number of model parameters to only three, all of which can be extracted from the atomistic MD simulation of short unentangled chains: (1) the Rouse time $\tau_{\mathrm{R}}$ was calculated from that of the shorter reference chains in atomistic MD

$$
\tau_{\mathrm{R}}=\tau_{\mathrm{R}, \text { ref }}\left(\frac{N}{N_{\text {ref }}}\right)^{2}
$$

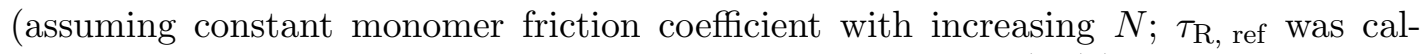
culated from the reference chain diffusivity through its MSD); (2) the entanglement strand length $N_{\mathrm{e}}$ (and plateau modulus via eq. (16)) - not directly available from short-chain simulations - was estimated using a scaling argument linking the tube diameter with the packing length [144], which eventually connects $N_{e}$ to the mean-square 
end-to-end distance $\left\langle R^{2}\right\rangle$

$$
\begin{gathered}
N_{\mathrm{e}}=\left(\frac{K}{\nu}\right)^{2}\left(\frac{N}{\left\langle R^{2}\right\rangle}\right)^{3} \\
\left\langle R^{2}\right\rangle=C_{\infty} N l_{\mathrm{b}}^{2}
\end{gathered}
$$

( $\nu=\rho / M_{0}$ is the number density of backbone bonds; $\rho$ is the density and $M_{0}$ the molecular weight per bond; $K$ is the prefactor in the scaling argument - empirically found from experimental data and constant at a given temperature for a wide variety of polymers; $l_{\mathrm{b}}$ is the backbone bond length; $C_{\infty}$ is the Flory's characteristic ratio estimated by extrapolating values of shorter chains from MD to the $N \rightarrow \infty$ limit); and (3) the glassy modulus $G_{\infty}$ is calculated from

$$
G_{\infty}=\left(\frac{\pi}{\sqrt{8}}\right) \nu k_{\mathrm{B}} T
$$

for which the high-frequency extension to the Rouse model by Marvin (which relates $G_{\infty}$ to the relaxation time in the glassy regime) $[145,146]$ and the relation by Benallal et al. [147] (which estimates the glassy relaxation time from $\tau_{\mathrm{R}}$ ) were invoked.

The atomistic benchmark system was a UA model for polybutadiene (PBD) with a microstructure that mixes cis-, trans-, and vinyl monomers [148]. The chain length was $\mathrm{C}_{114}$. Models (Davidson-Cole, Rouse, and tube) parameterized by this short-chain simulation were used to predict the linear viscoelasticity of a much longer chain $\mathrm{C}_{9140}$. For the reptation regime, the original Doi \& Edwards tube model [46] was compared with two later models that include CLF: modified Doi \& Edwards [149] and Milner \& McLeish [141] models. Comparison with experimental results showed that CLF is required to correctly capture the relaxation dynamics in the reptation regime and the Milner \& McLeish model provides the best overall agreement with experiments in both the plateau and terminal relaxation zones. In a follow-up study [91], they replaced the model predictions in the high-frequency (glassy and upper Rouse) regime with direct MD results (of the long chain system) using the Green-Kubo approach and integrated the MD profile with the Milner \& McLeish model prediction for lower frequencies. Excellent agreement with experiments was found (see fig. 6).

A more detailed approach has been developed by Stephanou, Mavrantzas, and coworkers that avoids the need of semi-empirical relations for tube model parameterization by directly retrieving the information from MD. It starts from the fundamental relation of any tube model that

$$
G(t)=G_{N}^{0} \Psi(t)
$$

where $\Psi(t)$ is the average fraction of the chain PP that is still contained in the original tube (of $t_{0}$ ) after a time period $t$ has elapsed. It is calculated from

$$
\Psi=\frac{1}{L} \int_{0}^{L} \psi(s, t) d s
$$

where $s$ is the arc-length coordinate of a chain segment along the contour of its PP and $\psi(s, t)$ is the segmental survival function - the probability that segment $s$ is still in the original tube after time $t$. Different tube models differ by the form of the 
(a)

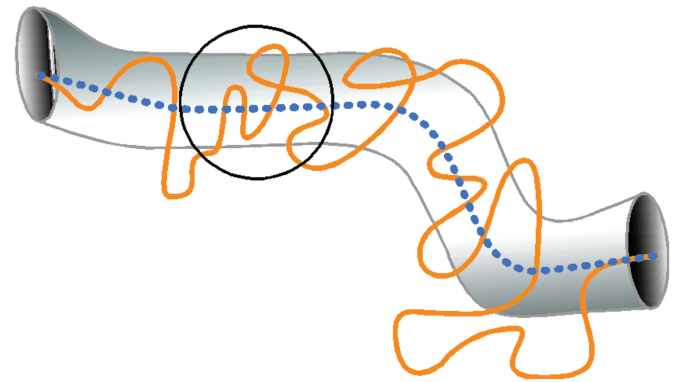

(b)

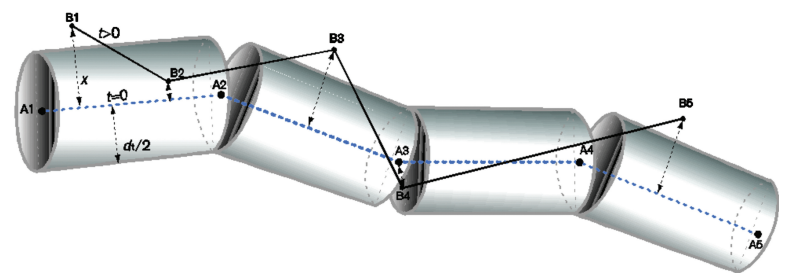

Figure 7. Schematics of (a) a polymer chain (solid/orange), its confining tube, and primitive path (PP; dashed/blue); and (b) a blowup view of a chain segment showing the reconstruction of the confining tube (cylinders) and the computation of the segmental survival function $\psi(s, t)$ using the Stephanou et al. [150] method. The blue/dashed line represents a small part of the PP at $t_{0}$. For each entanglement strand (segments between kinks), a cylinder of diameter $d_{\mathrm{T}}$ is constructed around its PP. After time $t$, the PP moves to new positions (solid/black line) and a fraction of it is found out of the original tube. Statistics of PP segments staying inside versus escaping the original tube are recorded to compute $\psi(s, t)$. (Reprinted from ref. [150], with the permission of AIP Publishing.)

$\psi(s, t)$ function, which, for the most part, describes the one-dimensional transport of segments along the tube contour. In the original Doi-Edwards model, an analytical expression for $\psi(s, t)$ is available [46]. More complex models describe the dynamics of $\psi(s, t)$ through a one-dimensional (along the PP contour) diffusion or reaction-diffusion partial differential equation (PDE).

Stephanou et al. [150] proposed a method that allows the direct computation of $\psi(s, t)$ from MD trajectories (fig. 7). It starts by applying the Z1 code [132] to convert the molecular configurations of each image into PP configurations. For $t_{0}$, the confining tube for each molecule is modeled as a piecewise cylindrical pipe around its PP with diameter $d_{\mathrm{T}}$ (computed either from the segmental MSD curve or the temporal $\mathrm{PP}$ fluctuations in the transverse directions). After time $t$, each PP segment is examined to check if it is still contained in the original tube. The probability of segment retention is averaged over all molecules and over different time origins. Applying this method to the atomistic MD data of weakly-entangled chains (this requirement differs from the earlier Byutner \& Smith approach where the benchmark simulation can be of unentangled chains) provides the direct MD measurement of $\psi(s, t)$ for the system. This $\psi(s, t)$ profile is used to parameterize the chosen tube model to match the model prediction (solution of the $\psi(s, t) \mathrm{PDE}$ ) with the MD result. At this step, the mathematical formulation of the model (the PDE problem) can be adjusted and improved to better describe the observed dynamics from MD, before it is used for prediction. The optimized model can then be solved for the $\psi(s, t)$ of much longer highly-entangled chains and their linear viscoelasticity can be predicted $[151,152]$. Invoking a few simple scaling relations can reduce the required model parameters to only the entanglement time $\tau_{\mathrm{e}}$ (available from the segmental MSD) and strand length $N_{\mathrm{e}}\left(N_{\mathrm{e}}^{\mathrm{Kuhn}}\right.$ calculated from the PP configurations was used [150]). The latter is again related to $G_{N}^{0}$ via eq. (16). 
The method has been applied to monodisperse polyethylene, cis-, and trans-1,4polybutadiene (PBD) [151] and for the latter two, bidisperse PBD was also studied [152]. For the Leygue et al. [153,154] tube model evaluated in both studies, improvements in the model formulation were proposed that led to better agreement in $\psi(s, t)$ between the model and MD. Comparison with experiments was also made with generally good agreement, although the prediction was found to be sensitive to model parameters and the revised tube models, which better predict $\psi(s, t)$, do not always improve the prediction of linear viscoelasticity [151]. This, however, could as well reflect the inadequacy of the models per se rather than that of the multiscale prediction strategy. The model prediction used for comparison with experiments in ref. [152] was not strictly $a b$ initio since tube model parameters were extracted from experiments rather than from atomistic simulation (because the experimental polymer contains both cis- and trans- monomers and thus does not exactly match the MD).

Another multiscale framework that connects atomistic MD, CGMD, tube theory, and continuum mechanics for the prediction of macroscopic viscoelastic behaviors of polymers was developed by Liu and coworkers $[106,133]$. Atomistic MD with the COMPASS (all-atom) force field [76] of short chains was used as the benchmark. In the case of cis-polyisoprene studied, a total of $n_{\text {chain }}=100$ chains, each with 10 repeating units, were used. The CG model maps each unit (4 backbone carbons) to one CG bead (superatom) using IBI. A time rescaling factor was determined by matching the velocity autocorrelation functions between the two model levels. The CG model was then used for the simulation of entangled chains from which PPs were analyzed using Kröger's Z1 code [132]. The CGMD and PP results were used to parameterize a constitutive model derived in their earlier work [155] based on the tube model and affine-deformation assumption, in which the stress tensor dynamics can be determined knowing the tube parameters (tube diameter, PP contour length, and Mittag-Leffler exponent - the last one was not systematically extracted and was set to unity in the study) and the chain statistics and dynamics from CGMD (Kuhn length, chain diffusivity, $\tau_{\mathrm{d}}$ ). Having a constitutive equation at the continuum level lifts the restriction of linear deformation - it can be solved for general nonlinear deformations (provided that changes in tube parameters under deformation are properly modeled) using the finite-element method. The approach was applied to polyethylene and polyisoprene with good agreements with experiments.

\subsubsection{Extending molecular simulation with slip-link/-spring models}

In slip-link models, topological constraints between entangled chains are conceptualized by analogy with a polymer network. Unlike a chemically crosslinked polymer, where the constraints are permanent and fixed, physical constraints between entangled chains are not attached to a particular atom or monomer in the chain sequence. Each such constraint is modeled as a slip link - a virtual ring that holds two chain segments together by restraining their lateral movement while still allowing them to wiggle their way out. The link is destroyed each time one of the chains escape the constraint and a new link is created when a chain's end reaches another chain. The idea was pioneered by Hua and Schieber [156] and a number of models of this type have been developed over the years $[157-160]$.

As a conceptual cousin to slip-link models, slip-spring models attach each link, which now only holds one chain, to an elastic spring and allow the links to move stochastically along the chain. Slip-spring models can be of a separate-chain type, in which the other end of the spring is attached to a fixed anchor point (see the Likhtman model [59] 


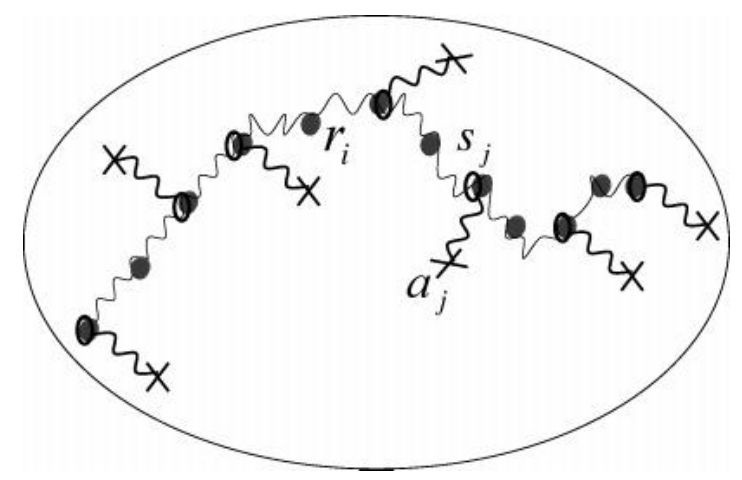

Figure 8. Schematic of the separate-chain slip-spring model of Likhtman [59]. Solid circles represents polymer beads $\left(\vec{r}_{i}\right)$ and light-colored springs are the backbone bonds. Dark-colored springs constrain slip-links (loops; $\vec{s}_{j}$ ) to fixed anchor points (crosses; $\vec{a}_{j}$ ). The slip-links move along the chain contour following stochastic dynamics. (Reprinted with permission from ref. [59]. Copyright (2005) American Chemical Society.)

in fig. 8 for example), or a multi-chain type, in which the other end of the spring is attached to another link, generally on a different chain - thus many chains must be simulated in the same cell (see the Theodorou model [161], illustrated in fig. 9, for example.) (It needs to be emphasized that the Theodorou model builds on a string of developments of multi-chain slip-spring models by a number of earlier researchers including Chappa et al. [162], Uneyama \& Masubuchi [163], Langeloth et al. [164], and the so-called TIEPOS scheme by Ramírez-Hernández et al. $[165,166]$. This particular model is singled out here because of its integration into a recent multiscale prediction framework that connects atomistic molecular models with entangled dynamics, which is discussed below.)

In addition to the slip-link vs. slip-spring and separate-chain vs. multi-chain distinctions, variations also exist between these models in other aspects, such as the specific rules for the movement, creation, and elimination of links. These models have been extensively discussed in a number of recent reviews [19-21]. The focus here is not on the models per se but on their application as an add-on tool for enhancing the capability of molecular simulation to predict the rheological properties of polymer.

Compared with tube models and earlier slip-link models, Likhtman's slip-spring model [59] stands out in its detailed treatment of sub-entanglement dynamics (i.e., at scales smaller than $N_{\mathrm{e}}$ ), which is important for weakly-entangled systems where the stress plateau is still not dominant. In the model (fig. 8), a polymer molecule is still described as a bead-spring chain. A number of links are added to different positions on the chain. Each link is constrained to an anchor point (fixed position in space) with a spring force, which effectively models the confinement of the chain segments around its PP with a parabolic potential. The links are allowed to slide along the chain contour with stochastic dynamics (i.e., under friction and random forces) to model the chain reptation in the tube - thus the word "slip" in its name. The links are positioned off-bead: i.e., they are allowed to take any position along the continuous arc-length coordinate of the chain contour. When a link falls between beads, interpolation is used to determine its spatial coordinates from its arc-length position. The link spring force, which tethers the link to its anchor point, is shared between the two neighboring beads. The model is solved numerically with BD for two sets of EoMs - one for the beads and the other for the links - and $G(t)$ can be calculated using the Green-Kubo relation.

The Likhtman model is a separate-chain model: an ensemble of isolated chains are 
simulated separately and each chain only directly interacts with its medium through slip-link spring forces. To include CR, which is fundamentally a multi-chain effect, coupling between chains is modeled by imposing binary correspondence between links. Each time a chain end moves past a link, that link and its corresponding link (on another chain) are both removed. A new pair of links, registered as a corresponding pair, are then added to the system: one at an end of a randomly-chosen chain, the other at a random position of another randomly-chosen chain.

There are three entanglement-related parameters: $N_{\mathrm{s}}$ - spring length, $N_{\mathrm{e}}$ - average number of backbone bonds between links, and $\zeta_{\mathrm{s}}$ - friction coefficient for the slip-links. It was noted that as long as $\zeta_{\mathrm{s}} \ll \zeta_{0}$ (friction coefficient of a polymer bead), results are not sensitive to its $\left(\zeta_{\mathrm{s}}\right)$ value. Meanwhile, for $N_{\mathrm{s}}$ and $N_{\mathrm{e}}$, it is their combination, rather than individual values, that is more important. Combinations that give the same $G_{N}^{0}$ predict very similar $G(t)$ profiles.

For the FENE bead-spring chain system, the Likhtman model has been used to predict the linear viscoelasticity of highly entangled polymers based on the simulation of weekly-entangled ones. As discussed in section 2.1.2, reliable computation of $G_{N}^{0}$ requires enormous chain lengths $(N \gtrsim O(1000))$ and system sizes. At $N=O(100)$, the chains are only moderately entangled and the reptation effect is still not dominant. Slip-link/-spring models were shown to better agree with experiments at $N \lesssim O(10) N_{\mathrm{e}}$ than tube models [167] and thanks to its better handling of sub-entanglement dynamics, the Likhtman model [59] is particularly suitable for extracting information from weakly-entangled simulation systems. This was later done by Likhtman et al. [54] using MD data for flexible FENE chains with $N$ up to 350 . Since $G_{N}^{0}$ is not available from the MD model of weakly-entangled chains, the slip-spring parameters (i.e., $N_{\mathrm{s}}$, $N_{\mathrm{e}}$, and $\zeta_{\mathrm{s}}$ ) were set at predetermined values based on those of common polymers. Instead, parameters of the FENE chain (size and time scale of individual beads) at the BD level (i.e. slip-spring simulation) were used as adjustable parameters to fit the $G(t)$ of weakly entangled chains from MD. Simulation of the slip-spring model was then run at the $N \rightarrow \infty$ limit to obtain the plateau modulus. The predicted $G_{N}^{0}$ was about $25 \%$ higher than the value from the step-strain elongation NEMD [47] (i.e., $N_{\mathrm{e}}$ lower than the latter).

More recently, a bottom-up hierarchical prediction framework was developed by Theodorou and coworkers [161] in an attempt to connect atomistic molecular models to the viscoelastic properties of highly-entangled polymer melts. The framework builds upon a slightly adapted version of a slip-spring model developed by the same group [168]. Different from the Likhtman model, the Theodorou slip-spring model is a multi-chain model: coupling between different chains is directly modeled by adding slip-springs between pairs of links on different chains (rather than between a link and a fixed anchor point). Therefore, all chains must be included in the same simulation cell to explicitly account for their interaction through slip-springs. For the slip-spring simulation, the CG bead-spring chain model for polymers is still updated with $\mathrm{BD}$, but the slip-link positions are updated with a kinetic Monte Carlo (kMC) approach. Link positions are on-bead - links can only connect to a discrete number of positions along the chain marked by the polymer beads - and they are allowed to hop between nearby beads through Monte Carlo sampling.

Mapping between the atomistic and BD models followed a systematic CG approach. $\mathrm{CG}$ potentials were constructed from the atomistic benchmark with $\mathrm{BI}$ and the friction coefficient of the BD beads was determined by scaling up that of the backbone $\mathrm{CH}_{2}$ 


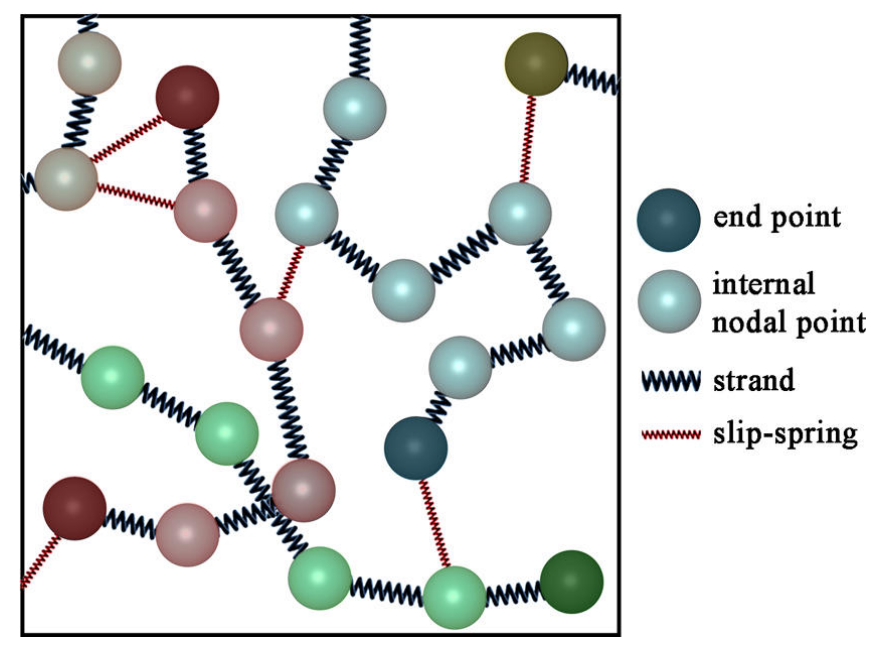

Figure 9. Schematic of a multi-chain slip-spring model of Theodorou et al. [168]. Thicker springs present backbone bonds and thinner springs represent slip-spring potentials between links (not explicitly drawn) on different chains. Links hop between beads following a kinetic Monte Carlo algorithm. (Reprinted with permission from ref. [161]. Copyright (2017) American Chemical Society.)

group at the atomistic level

$$
\zeta_{\mathrm{BD}, 0}=\frac{m_{\mathrm{BD}, 0}}{m_{\mathrm{MD}, 0}} \zeta_{\mathrm{MD}, 0}
$$

where $\zeta$ and $m$ are the friction coefficient and molecular mass, respectively, and the subscript 0 indicates individual repeating units (bead in $\mathrm{BD}$ and $\mathrm{CH}_{2}$ in $\mathrm{MD}$ ). Singleunit friction coefficient $\zeta_{\mathrm{MD}, 0}$ can be straightforwardly obtained from MD (from the relaxation time of the smallest Rouse mode using the Stokes-Einstein law) [128]. Determination of the slip-spring settings was less methodical (not entirely ab initio - i.e., from atomistic benchmark), but microscopic insight was still taken into account in most choices. The spring potential has a soft FENE form and the potential parameters were determined to match the spring-length distribution to $d_{\mathrm{T}}$ of the highly-entangled polymer. (Because of the softness of the interaction, free energy contribution from these artificially-added interactions is minimized. In the case of dense and stiff slip links, an expensive free energy compensation term proposed by Chappa et al. [162] would be required.) The average spacing between consecutive slip-links along the chain was chosen within the range from the $N_{\mathrm{e}}$ measured with the CReTA algorithm [136] to the experimental value (the latter is 2.5 times as large as the former, which is consistent with the aforementioned analysis by Everaers [139] that the experimental value is close to $N_{\mathrm{e}}^{\mathrm{Kuhn}}$ and $N_{\mathrm{e}}^{\mathrm{Kuhn}} \approx 2 N_{\mathrm{e}}^{\mathrm{indv}}$ ) and adjusted to match the experimental plateau modulus. Finally, the slip-link hopping frequency was mostly an adjustable parameter set to match the chain diffusivity from $\mathrm{BD} / \mathrm{kMC}$ to that in atomistic MD. For rheological prediction, the Green-Kubo relation was used to calculate $G(t)$ and the multiple-tau correlator algorithm [62] was invoked for SAF computation and smoothing. Maxwell modes were used to fit the long-time region of $G(t)$ (which was also used in ref. [54] to get a smooth profile for calculating $G^{*}(\omega)$ using eqs. (14) and (15)).

In ref. [161], UA models of entangled polyethylene with $\mathrm{C}_{260}$ and $\mathrm{C}_{520}$ were used as the atomistic benchmark cases and the $\mathrm{BD} / \mathrm{kMC}$ model was built for up to $\mathrm{C}_{2080}$. Both equilibrium and non-equilibrium dynamics were run and extrapolation of the shear viscosity from non-equilibrium $\mathrm{BD}$ to the zero-shear limit matches with the 
Green-Kubo result.

\section{Summary and outlook}

This review article covers the development of molecular simulation as a tool for the prediction of polymer rheological properties. Despite much progress made over more than two decades, challenges still persist especially in two aspects: (1) large uncertainty caused by the strong stress fluctuations in microscopic simulation cells and (2) intractable time scales for the relaxation of polymer chains, especially in the entangled regime. Direct prediction by molecular dynamics can take either equilibrium or non-equilibrium approaches. Results from EMD and NEMD have been found to agree well with one another. The main challenge is to obtain statistically reliable results, for the whole spectrum of linear viscoelasticity, with reasonable computational time. For atomistic molecular models, the computational cost is higher and stress fluctuations intensify - direct MD prediction of the linear viscoelastic behaviors for long chain polymers remains out of reach.

Mitigating the impact of stress fluctuations requires large simulation system sizes and noise-filtering techniques. (For molecular liquids described by atomistic models, combination of multiple noise-filtering techniques is often required.) Systematic coarsegraining approaches can be used for addressing the time scale challenge, provided that information on system dynamics, in addition to that of the molecular structure, is properly pipelined from the atomistic simulation to the CG model. Advancement in rheological modeling provides an alternative direction to tackle these challenges. For unentangled chains, the Rouse model has been used to extract the necessary information from molecular simulation for reconstructing the dynamics. The stress autocorrelation function is typically underestimated because of the neglect of intermolecular interactions - for which a compensation term can be extracted from the short-time dynamics in MD (see ref. [44]) - as well as other simplifications in the model. For entangled polymers, various forms of tube and slip-link/-spring models have been developed. Molecular simulation can be integrated with such models to make chemically-specific predictions for long-chain polymers, by systematically extracting model parameters from the atomistic simulation.

The ultimate objective is a systematic workflow that connects materials chemistry - including monomer chemistry, molecular architecture, molecular weight distribution, and, in the case of mixtures, composition - with experimentally verifiable rheological properties. The prediction will be ab initio in the sense that information is extracted from the detailed molecular models and conveyed, in a bottom-up manner, to higher-level models for macroscopic prediction. Several such multiscale integrated frameworks have already been demonstrated for highly-entangled polymers. These models either adopt a systematic CG paradigm and impose inter-chain topological constraints by explicitly modeling the entanglements between bonds [22,122-124] or integrate atomistic molecular models with tube or slip-link/-spring models (and sometimes a CG model in between) to extend the dynamics from unentangled or, more often, weakly-entangled chains (from MD) to highly-entangled systems comparable to experiments $[91,106,133,142,150-152,161]$. The level of success reached so far gives us reasons to be optimistic: reliable ab initio prediction of polymer rheology for industrially-relevant polymer systems is in sight.

The current review is obviously not all-inclusive. Much emphasis has been put on linear viscoelasticity and, for nonlinear rheology, only the steady-shear viscosity was 
discussed in more detail. These quantities are not only most frequently studied in molecular simulation, but also most often measured in experiments for standard rheological characterizations. The review also focuses on the prediction of quantitative rheological properties and has skipped many contributions using simulation for the fundamental understanding of rheological responses and polymer dynamics. For example, studies such as those using NEMD to reveal chain conformation and dynamics in non-linear flows are not covered $[41,85,169,170]$. Extensional rheology has also been largely left out, because NEMD simulation of the large-strain regime was limited to planar extensional flow [170] until fairly recently. In particular, the development of a generalized Kraynik-Reinelt boundary condition finally enabled the molecular simulation of three-dimensional flows such as uniaxial and biaxial extensions [171], making the prediction of non-linear extensional rheology a tangible possibility [172].

As to the polymers being studied, much of the focus has been put on monodisperse linear polymer melts. Extension to polymers with other molecular architectures or realistic molecular weight distributions (MWDs) seems to be straightforward as far as molecular simulation is concerned. The challenge is to develop reliable rheological models for such systems. Modeling the dynamics and rheology of entangled polydisperse polymer melts typically involves a mixing rule between the relaxation spectra of different chain lengths $[173,174]$ (which was considered in the Leygue et al. tube model $[153,154]$ used for bidisperse polybutadiene in ref. [152]). Meanwhile, Cao and Likhtman [127] have demonstrated, using the MD simulation of relatively short FENE bead-spring chains ( $N=10$ and 100), that the cross-correlation between the relaxation dynamics of different chains (of either identical or different chain lengths) takes up the same proportion of the overall relaxation of the system, for both monodisperse and bidisperse systems of different blend compositions. This finding opens the possibility for extending the MD prediction from monodisperse systems to general polydisperse ones. Extending the tube and slip-link models to branched and other complex chain architectures has been an ongoing progress [19-21,175,176].

Challenges in applying molecular simulation for industrially-relevant polymers also come from the experimental side. Take low-density polyethylene (LDPE) for example. The vast distribution of chain sizes and branching configurations in commercial LDPE samples makes the experimental characterization of detailed molecular structures nearly impossible. Rheological prediction of such materials must be made based on incomplete information, which appears to be an insurmountable obstacle. Read et al. [177] sidestepped this problem by invoking the Tobita model for the kinetics of homogeneous free radical polymerization [178] to model the the branching configuration distribution of LDPE. Parameters for the Tobita model were obtained by fitting with the experimentally measured MWD and the radius of gyration of the molecules. Tube model parameters were fitted with the linear viscoelastic data. Prediction of non-linear shear and extensional rheology using the same parameters (with no further adjustment) successfully captured the experimental results. Although molecular simulation was not involved in that study, the idea of integrating polymerization kinetics into the modeling of complex industrial polymers provides new inspirations for the future development of more comprehensive predictive frameworks based on molecular simulation.

Finally, the author's own interest in this area is motivated by a larger effort in his research group aiming at incorporating molecular simulation into the toolbox for the selection and design of industrial plasticizers. Plasticizers are particularly important for the production of poly(vinyl chloride) (PVC) materials. Classical theories for explaining the plasticization effect lacks in molecular details $[179,180]$ and can- 
not provide chemically-specific guidelines. Therefore, a priori prediction of plasticizer performance is of great interest and can potentially be used to reduce the time and cost of experimental development. In a recent study from the initiative, a reliable atomistic molecular model has been developed for the prediction of thermo-physical and mechanical properties of plasticized PVC [181]. Rheological prediction is part of the ongoing effort. Extending the existing methods covered in this review to polymerplasticizer blends will be a new test to the models themselves. Compared with the relatively better-known (but still not widely studied) case of polydisperse polymers, the polymer-plasticizer blends have an additional layer of complexity in the specific interactions between the additive and the host polymer. A better understanding of how these interactions affect rheology is also a key question to address for the fundamental molecular understanding of the plasticization effect.

\section{Acknowledgement(s)}

Financial support from the Natural Sciences and Engineering Research Council (NSERC) of Canada (RGPIN-4903-2014, CRDPJ-514051-17) is acknowledged. The author would also like to thank Ph.D. students Oluseye Adeyemi and Ali Heydari Beni for their careful review of the manuscript and suggestions.

\section{References}

[1] Yan D, Wang WJ, Zhu S. Effect of long chain branching on rheological properties of metallocene polyethylene. Polymer. 1999;40:1737-1744.

[2] Glotzer SC, Paul W. Molecular and mesoscale simulation methods for polymer materials. Annu Rev Mater Res. 2002;32:401-436.

[3] Paul W, Smith GD. Structure and dynamics of amorphous polymers: Computer simulations compared to experiment and theory. Rep Prog Phys. 2004;67:1117-1185.

[4] Theodorou DN, Suter UW. Detailed molecular-structure of a vinyl polymer glass. Macromolecules. 1985;18:1467-1478.

[5] Mavrantzas VG, Boone TD, Zervopoulou E, et al. End-bridging Monte Carlo: A fast algorithm for atomistic simulation of condensed phases of long polymer chains. Macromolecules. 1999;32:5072-5096.

[6] Auhl R, Everaers R, Grest GS, et al. Equilibration of long chain polymer melts in computer simulations. J Chem Phys. 2003;119:12718-12728.

[7] Carbone P, Karimi-Varzaneh HA, Müller-Plathe F. Fine-graining without coarsegraining: an easy and fast way to equilibrate dense polymer melts. Faraday Discuss. 2010;144:25-42.

[8] Zhang G, Moreira LA, Stuehn T, et al. Equilibration of high molecular weight polymer melts: A hierarchical strategy. ACS Macro Lett. 2014;3:198-203.

[9] Sliozberg YR, Kröger M, Chantawansri TL. Fast equilibration protocol for million atom systems of highly entangled linear polyethylene chains. J Chem Phys. 2016;144:154901.

[10] Müller-Plathe F, CRogers S, van Gunsteren WF. Computational evidence for anomalous diffusion of small molecules in amorphous polymers. Chem Phys Lett. 1992;199:237-243.

[11] Sok RM, Berendsen HJC, van Gunsteren WF. Molecular-dynamics simulation of the transport of small molecules across a polymer membrane. J Chem Phys. 1992;96:46994704.

[12] Charati SG, Stern SA. Diffusion of gases in silicone polymers: Molecular dynamics simulations. Macromolecules. 1998;31:5529-5535.

[13] Greenfield ML, Theodorou DN. Molecular modeling of methane diffusion in glassy at- 
actic polypropylene via multidimensional transition state theory. Macromolecules. 1998; 31:7068-7090.

[14] Greenfield ML, Theodorou DN. Coarse-grained molecular simulation of penetrant diffusion in a glassy polymer using reverse, and kinetic Monte Carlo. Macromolecules. 2001; 34:8541-8553.

[15] Hanson B, Pryamitsyn V, Ganesan V. Computer simulations of gas diffusion in polystyrene- $\mathrm{C}_{60}$ fullerene nanocomposites using trajectory extending kinetic Monte Carlo method. J Phys Chem B. 2012;116:95-103.

[16] Xi L, Shah M, Trout BL. Hopping of water in a glassy polymer studied via transition path sampling and likelihood maximization. J Phys Chem B. 2013;117:3634-3647.

[17] Watanabe H. Viscoelasticity and dynamics of entangled polymers. Prog Polym Sci. 1999; 24:1253-1403.

[18] McLeish TCB. Tube theory of entangled polymer dynamics. Adv Phys. 2002;51:13791527.

[19] Larson RG, Zhou Q, Shanbhag S, et al. Advances in modeling of polymer melt rheology. AIChE J. 2007;53:542-548.

[20] Masubuchi Y. Simulating the flow of entangled polymers. Annu Rev Chem Biomol Eng. $2014 ; 5: 11-33$.

[21] Schieber JD, Andreev M. Entangled polymer dynamics in equilibrium and flow modeled through slip links. Annu Rev Chem Biomol Eng. 2014;5:367-381.

[22] Padding JT, Briels WJ. Systematic coarse-graining of the dynamics of entangled polymer melts: The road from chemistry to rheology. J Phys-Condes Matter. 2011;23:233101.

[23] Kremer K, Grest G. Dynamics of entangled linear polymer melts: A molecular-dynamics simulation. J Chem Phys. 1990;92:5057-5086.

[24] Allen MP, Tildesley DJ. Computer Simulation of Liquids. 2nd ed. New York: Oxford University Press; 2017.

[25] Bird RB, Curtis CF, Armstrong RC, et al. Dynamics of polymeric liquids. 2nd ed. Vol. 2. New York: John Wiley \& Sons; 1987.

[26] Graham MD. Microhydrodynamics, Brownian Motion, and Complex Fluids. Cambridge: Cambridge University Press; 2018.

[27] Kröger M. Simple models for complex nonequilibrium fluids. Phys Rep. 2004;390:453551.

[28] Takahashi KZ, Nishimura R, Yamato N, et al. Onset of static and dynamic universality among molecular models of polymers. Sci Rep. 2017;7:12379.

[29] Zhou Q, Larson RG. Direct molecular dynamics simulation of branch point motion in asymmetric star polymer melts. Macromolecules. 2007;40:3443-3449.

[30] Duering ER, Kremer K, Grest GS. Structure and relaxation of end-linked polymer networks. J Chem Phys. 1994;101:8169-8192.

[31] Zhang S, Xi L. Effects of precursor topology on polymer networks simulated with molecular dynamics. Polymer. 2017;116:143-152.

[32] Halverson JD, Lee WB, Grest GS, et al. Molecular dynamics simulation study of nonconcatenated ring polymers in a melt. I. Statics. J Chem Phys. 2011;134:204904.

[33] Evans DJ, Morriss GP. Nonlinear-response theory for steady planar Couette flow. Phys Rev A. 1984;30:1528-1530.

[34] Lees A, Edwards S. The computer study of transport processes under extreme conditions. J Phys C: Solid State Phys. 1972;5:1921-1929.

[35] Todd BD, Daivis PJ. Homogeneous non-equilibrium molecular dynamics simulations of viscous flow: Techniques and applications. Mol Simulat. 2007;33:189-229.

[36] Kröger M, Loose W, Hess S. Rheology and structural changes of polymer melts via nonequilibrium molecular dynamics. J Rheol. 1993;37:1057-1079.

[37] Xu Z, de Pablo JJ, Kim S. Transport properties of polymer melts from nonequilibrium molecular dynamics. J Chem Phys. 1995;102:5836-5844.

[38] Kröger M, Hess S. Rheological evidence for a dynamical crossover in polymer melts via nonequilibrium molecular dynamics. Phys Rev Lett. 2000;85:1128-1131. 
[39] Aoyagi T, Doi M. Molecular dynamics simulation of entangled polymers in shear flow. Comput Theor Polym Sci. 2000;10:317-321.

[40] Bird RB, Armstrong RC, Hassager O. Dynamics of polymeric liquids. 2nd ed. Vol. 1. New York: John Wiley \& Sons; 1987.

[41] Cao J, Likhtman AE. Simulating startup shear of entangled polymer melts. ACS Macro Lett. 2015;4:1376-1381.

[42] Rubinstein M, Colby RH. Polymer physics. New York: Oxford University Press; 2003.

[43] Hernández Cifre JG, Hess S, Kröger M. Linear viscoelastic behavior of unentangled polymer melts via non-equilibrium molecular dynamics. Macromol Theory Simul. 2004; $13: 748-753$.

[44] Vladkov M, Barrat JL. Linear and nonlinear viscoelasticity of a model unentangled polymer melt: Molecular dynamics and rouse modes analysis. Macromol Theory Simul. 2006;15:252-262.

[45] Allen MP, Tildesley DJ. Computer Simulation of Liquids. New York: Oxford University Press; 1989.

[46] Doi M, Edwards SF. The theory of polymer dynamics. New York: Oxford University Press; 1986.

[47] Pütz M, Kremer K, Grest GS. What is the entanglement length in a polymer melt? Europhys Lett. 2000;49:735-741.

[48] Everaers R, Sukumaran SK, Grest GS, et al. Rheology and microscopic topology of entangled polymeric liquids. Science. 2004;303:823-826.

[49] Hsu HP, Kremer K. Static and dynamic properties of large polymer melts in equilibrium. J Chem Phys. 2016;144:154907.

[50] Tuckerman ME. Statistical Mechanics: Theory and Molecular Simulation. New York: Oxford University Press; 2010.

[51] Xu Z, Khare R, de Pablo JJ, et al. On the calculation of transport properties of polymer melts from nonequilibrium molecular dynamics. J Chem Phys. 1997;106:8285-8286.

[52] Sen S, Kumar SK, Keblinski P. Viscoelastic properties of polymer melts from equilibrium molecular dynamics simulations. Macromolecules. 2005;38:650-653.

[53] Zhou Q, Larson RG. Direct calculation of the tube potential confining entangled polymers. Macromolecules. 2006;39:6737-6743.

[54] Likhtman AE, Sukumaran SK, Ramírez J. Linear viscoelasticity from molecular dynamics simulation of entangled polymers. Macromolecules. 2007;40:6748-6757.

[55] Lee WB, Kremer K. Entangled polymer melts: Relation between plateau modulus and stress autocorrelation function. Macromolecules. 2009;42:6270-6276.

[56] Masubuchi Y, Uneyama T. Comparison among multi-chain models for entangled polymer dynamics. Soft Matter. 2018;14:5986-5994.

[57] Likhtman AE, Sukumaran SK. Comment on "Entangled polymer melts: Relation between plateau modulus and stress autocorrelation function". Macromolecules. 2010; 43:3980-3983.

[58] Lee WB, Halverson J, Kremer K. Reply to comment on "Entangled polymer melts: Relation between plateau modulus and stress autocorrelation function. Macromolecules. 2010;43:3984-3985.

[59] Likhtman AE. Single-chain slip-link model of entangled polymers: Simultaneous description of neutron spin-echo, rheology, and diffusion. Macromolecules. 2005;38:6128-6139.

[60] Dealy JM, Larson RG. Structure and rheology of molten polymers: From structure to flow behavior and back again. Munich: Carl Hanser Verlag; 2006.

[61] Sen S, Kumar SK, Keblinski P. Analysis of uncertainties in polymer viscoelastic properties obtained from equilibrium computer simulations. J Chem Phys. 2006;124:144909.

[62] Ramírez J, Sukumaran SK, Vorselaars B, et al. Efficient on the fly calculation of time correlation functions in computer simulations. J Chem Phys. 2010;133:154103.

[63] Magatti D, Ferri F. Fast multi-tau real-time software correlator for dynamic light scattering. Appl Optics. 2001;40:4011-4021.

[64] Mason T, Ganesan K, van Zanten J, et al. Particle tracking microrheology of complex 
fluids. Phys Rev Lett. 1997;79:3282-3285.

[65] Squires TM, Mason TG. Fluid mechanics of microrheology. Annu Rev Fluid Mech. 2010; 42:413-438.

[66] Karim M, Kohale SC, Indei T, et al. Determination of viscoelastic properties by analysis of probe-particle motion in molecular simulations. Phys Rev E. 2012;86:051501.

[67] Kuhnhold A, Paul W. Passive one-particle microrheology of an unentangled polymer melt studied by molecular dynamics simulation. Phys Rev E. 2014;90:022602.

[68] Kuhnhold A, Paul W. Active one-particle microrheology of an unentangled polymer melt studied by molecular dynamics simulation. Phys Rev E. 2015;91:042601.

[69] Karim M, Indei T, Schieber JD, et al. Determination of linear viscoelastic properties of an entangled polymer melt by probe rheology simulations. Phys Rev E. 2016;93:012501.

[70] Indei T, Schieber JD, Cordoba A, et al. Treating inertia in passive microbead rheology. Phys Rev E. 2012;85:021504.

[71] Indei T, Schieber JD, Cordoba A. Competing effects of particle and medium inertia on particle diffusion in viscoelastic materials, and their ramifications for passive microrheology. Phys Rev E. 2012;85:041504.

[72] Liu J, Cao D, Zhang L. Molecular dynamics study on nanoparticle diffusion in polymer melts: A test of the stokes-einstein law. J Phys Chem C. 2008;112:6653-6661.

[73] Yamamoto U, Schweizer KS. Theory of nanoparticle diffusion in unentangled and entangled polymer melts. J Chem Phys. 2011;135:224902.

[74] Maple JR, Hwang MJ, Stockfisch TP, et al. Derivation of class-II force-fields .1. Methodology and quantum force-field for the alkyl functional-group and alkane molecules. J Comput Chem. 1994;15:162-182.

[75] Jorgensen WL, Maxwell DS, Tirado-Rives J. Development and testing of the OPLS allatom force field on conformational energetics and properties of organic liquids. J Am Chem Soc. 1996;118:11225-11236.

[76] Sun H. COMPASS: An ab initio force-field optimized for condensed-phase applications - Overview with details on alkane and benzene compounds. J Phys Chem B. 1998; 102:7338-7364.

[77] Martin MG, Siepmann J. Transferable potentials for phase equilibria. 1. United-atom description of n-alkanes. J Phys Chem B. 1998;102:2569-2577.

[78] Cummings PT, Evans DJ. Nonequilibrium molecular dynamics approaches to transport properties and non-newtonian fluid rheology. Ind Eng Chem Res. 1992;31:1237-1252.

[79] Hess B. Determining the shear viscosity of model liquids from molecular dynamics simulations. J Chem Phys. 2002;116:209.

[80] Mark P, Nilsson L. Structure and dynamics of the TIP3P, SPC, and SPC/E water models at 298 K. J Phys Chem B. 2001;105:9954-9960.

[81] Daivis PJ, Evans DJ. Comparison of constant pressure and constant volume nonequilibrium simulations of sheared model decane. J Chem Phys. 1994;100:541-547.

[82] Mondello M, Grest GS. Viscosity calculations of n-alkanes by equilibrium molecular dynamics. J Chem Phys. 1997;106:9327.

[83] Tobias D, Tu K, Klein M. Assessment of all-atom potentials for modeling membranes: molecular dynamics simulations of solid and liquid alkanes and crystals of phospholipid fragments. J Chim Phys Phys-Chim Biol. 1997;94:1482-1502. CECAM Meeting on Potential Functions for Simulation of Biomolecular Systems, LYON, FRANCE, SEP 23-25, 1996.

[84] Payal RS, Balasubramanian S, Rudra I, et al. Shear viscosity of linear alkanes through molecular simulations: quantitative tests for $\mathrm{n}$-decane and $\mathrm{n}$-hexadecane. Mol Simul. 2012;38:1234-1241.

[85] Baig C, Mavrantzas VG, Kroger M. Flow effects on melt structure and entanglement network of linear polymers: results from a nonequilibrium molecular dynamics simulation study of a polyethylene melt in steady shear. Macromolecules. 2010;43:6886-6902.

[86] Cho S, Jeong S, Kim JM, et al. Molecular dynamics for linear polymer melts in bulk and confined systems under shear flow. Sci Rep. 2017;7:9004. 
[87] Zhang Y, Otani A, Maginn EJ. Reliable viscosity calculation from equilibrium molecular dynamics simulations: a time decomposition method. J Chem Theory Comput. 2015; 11:3537-3546.

[88] Chen T, Smit B, Bell AT. Are pressure fluctuation-based equilibrium methods really worse than nonequilibrium methods for calculating viscosities? J Chem Phys. 2009; 131:246101.

[89] Rey-Castro C, Vega LF. Transport properties of the ionic liquid 1-ethyl-3methylimidazolium chloride from equilibrium molecular dynamics simulation. The effect of temperature. J Phys Chem B. 2006;110:14426-14435.

[90] Harmandaris VA, Mavrantzas VG, Theodorou DN. Atomistic molecular dynamics simulation of stress relaxation upon cessation of steady-state uniaxial elongational flow. Macromolecules. 2000;33:8062-8076.

[91] Byutner O, Smith GD. Viscoelastic properties of polybutadiene in the glassy regime from molecular dynamic simulations. Macromolecules. 2002;35:3769-3771.

[92] Zhang L, Greenfield ML. Analyzing properties of model asphalts using molecular simulation. Energy Fuels. 2007;21:1712-1716.

[93] Zhang L, Greenfield ML. Relaxation time, diffusion, and viscosity analysis of model asphalt systems using molecular simulation. J Chem Phys. 2007;127:194502.

[94] Li DD, Greenfield ML. Chemical compositions of improved model asphalt systems for molecular simulations. Fuel. 2014;115:347-356.

[95] Khabaz F, Khare R. Molecular simulations of asphalt rheology: Application of timetemperature superposition principle. J Rheol. 2018;62:941-954.

[96] Williams ML, Landel RF, Ferry JD. The temperature dependence of relaxation mechanisms in amorphous polymers and other glass-forming liquids. J Am Chem Soc. 1955; 77:3701-3707.

[97] Masoori M, Greenfield ML. Reducing noise in computed correlation functions using techniques from signal processing. Mol Simul. 2017;43:1485-1495.

[98] Harmandaris VA, Kremer K. Dynamics of polystyrene melts through hierarchical multiscale simulations. Macromolecules. 2009;42:791-802.

[99] Kremer K, Müller-Plathe F. Multiscale problems in polymer science: simulation approaches. MRS Bull. 2001;26:205-210.

[100] Marrink SJ, Risselada HJ, Yefimov S, et al. The MARTINI force field: Coarse grained model for biomolecular simulations. J Phys Chem B. 2007;111:7812-7824.

[101] Muller-Plathe F. Coarse-graining in polymer simulation: From the atomistic to the mesoscopic scale and back. ChemPhysChem. 2002;3:754-769.

[102] Ayton GS, Noid WG, Voth GA. Multiscale modeling of biomolecular systems: in serial and in parallel. Curr Opin Struct Biol. 2007;17:192-198.

[103] Peter C, Kremer K. Multiscale simulation of soft matter systems - from the atomistic to the coarse-grained level and back. Soft Matter. 2009;5(22):4357-4366.

[104] Rüehle V, Junghans C, Lukyanov A, et al. Versatile object-oriented toolkit for coarsegraining applications. J Chem Theory Comput. 2009;5:3211-3223.

[105] Brini E, Algaer EA, Ganguly P, et al. Systematic coarse-graining methods for soft matter simulations-a review. Soft Matter. 2013;9:2108-2119.

[106] Li Y, Abberton BC, Kröger M, et al. Challenges in multiscale modeling of polymer dynamics. Polymers. 2013;5:751-832.

[107] Ganguly P, Mukherji D, Junghans C, et al. Kirkwood-Buff coarse-grained force fields for aqueous solutions. J Chem Theory Comput. 2012;8:1802-1807.

[108] Izvekov S, Voth GA. Multiscale coarse graining of liquid-state systems. J Chem Phys. $2005 ; 123: 134105$.

[109] Noid WG, Chu J, Ayton GS, et al. The multiscale coarse-graining method. I. A rigorous bridge between atomistic and coarse-grained models. J Chem Phys. 2008;128:244114.

[110] Fritz D, Koschke K, Harmandaris VA, et al. Multiscale modeling of soft matter: Scaling of dynamics. Phys Chem Chem Phys. 2011;13:10412-10420.

[111] Depa P, Chen C, Maranas JK. Why are coarse-grained force fields too fast? A look at 
dynamics of four coarse-grained polymers. J Chem Phys. 2011;134:014903.

[112] Harmandaris VA, Adhikari NP, van der Vegt NFA, et al. Hierarchical modeling of polystyrene: From atomistic to coarse-grained simulations. Macromolecules. 2006; 39:6708-6719.

[113] Harmandaris VA, Kremer K. Predicting polymer dynamics at multiple length and time scales. Soft Matter. 2009;5:3920-3926.

[114] Groot RD, Warren PB. Dissipative particle dynamics: Bridging the gap between atomistic and mesoscopic simulation. J Chem Phys. 1997;107:4423-4435.

[115] Ermak DL, McCammon JA. Brownian dynamics with hydrodynamic interactions. J Chem Phys. 1978;69:1352-1360.

[116] Maurel G, Schnell B, Goujon F, et al. Multiscale modeling approach toward the prediction of viscoelastic properties of polymers. J Chem Theory Comput. 2012;8:4570-4579.

[117] Reith D, Pütz M, Müller-Plathe F. Deriving effective mesoscale potentials from atomistic simulations. J Comput Chem. 2003;24:1624-1636.

[118] Shell MS. Systematic coarse-graining of potential energy landscapes and dynamics in liquids. J Chem Phys. 2012;137:084503.

[119] Shell MS. The relative entropy is fundamental to multiscale and inverse thermodynamic problems. J Chem Phys. 2008;129:144108.

[120] Izvekov S, Voth GA. Modeling real dynamics in the coarse-grained representation of condensed phase systems. J Chem Phys. 2006;125:151101.

[121] Markutsya S, Lamm MH. A coarse-graining approach for molecular simulation that retains the dynamics of the all-atom reference system by implementing hydrodynamic interactions. J Chem Phys. 2014;141:174107.

[122] Padding JT, Briels WJ. Uncrossability constraints in mesoscopic polymer melt simulations: Non-rouse behavior of C120H242. J Chem Phys. 2001;115:2846-2859.

[123] Padding JT, Briels WJ. Time and length scales of polymer melts studied by coarsegrained molecular dynamics simulations. J Chem Phys. 2002;117:925-943.

[124] Padding JT, Briels WJ. Coarse-grained molecular dynamics simulations of polymer melts in transient and steady shear flow. J Chem Phys. 2003;118:10276-10286.

[125] Kumar S, Larson RG. Brownian dynamics simulations of flexible polymers with springspring repulsions. J Chem Phys. 2001;114:6937-6941.

[126] Goujon F, Malfreyt P, Tildesley DJ. Mesoscopic simulation of entanglements using dissipative particle dynamics: Application to polymer brushes. J Chem Phys. 2008; 129:034902.

[127] Cao J, Likhtman AE. Time-dependent orientation coupling in equilibrium polymer melts. Phys Rev Lett. 2010;104:207801.

[128] Harmandaris VA, Mavrantzas VG, Theodorou DN. Atomistic molecular dynamics simulation of polydisperse linear polyethylene melts. Macromolecules. 1998;31:7934-7943.

[129] Masubuchi Y, Takata H, Amamoto Y, et al. Relaxation of Rouse modes for unentangled polymers obtained by molecular simulations. Nihon Reoroji Gakkaishi. 2018;46:171-178.

[130] Edwards S. The statistical mechanics of polymerized material. Proc Phys Soc. 1967;92:9.

[131] de Gennes PG. Reptation of a polymer chain in the presence of fixed obstacles. J Chem Phys. 1971;55:572-579.

[132] Kröger M. Shortest multiple disconnected path for the analysis of entanglements in twoand three-dimensional polymeric systems. Comput Phys Commun. 2005;168:209-232.

[133] Li Y, Tang S, Abberton BC, et al. A predictive multiscale computational framework for viscoelastic properties of linear polymers. Polymer. 2012;53:5935-5952.

[134] Edwards S. The theory of rubber elasticity. Br Polym J. 1977;9:140-143.

[135] Sukumaran SK, Grest GS, Kremer K, et al. Identifying the primitive path mesh in entangled polymer liquids. J Polym Sci Pt B-Polym Phys. 2005;43:917-933.

[136] Tzoumanekas C, Theodorou DN. Topological analysis of linear polymer melts: A statistical approach. Macromolecules. 2006;39:4592-4604.

[137] Shanbhag S, Kröger M. Primitive path networks generated by annealing and geometrical methods: Insights into differences. Macromolecules. 2007;40:2897-2903. 
[138] Shanbhag S, Larson R. Identification of topological constraints in entangled polymer melts using the bond-fluctuation model. Macromolecules. 2006;39:2413-2417.

[139] Everaers R. Topological versus rheological entanglement length in primitive-path analysis protocols, tube models, and slip-link models. Phys Rev E. 2012;86:022801.

[140] Tzoumanekas C, Theodorou DN. From atomistic simulations to slip-link models of entangled polymer melts: Hierarchical strategies for the prediction of rheological properties. Curr Opin Solid State Mater Sci. 2006;10(2):61-72.

[141] Milner S, McLeish T. Reptation and contour-length fluctuations in melts of linear polymers. Phys Rev Lett. 1998;81:725-728.

[142] Byutner O, Smith GD. Prediction of the linear viscoelastic shear modulus of an entangled polybutadiene melt from simulation and theory. Macromolecules. 2001;34:134-139.

[143] Davidson DW, Cole RH. Dielectric relaxation in glycerol, propylene glycol, and npropanol. J Chem Phys. 1951;19:1484-1490.

[144] Fetters LJ, Lohse DJ, Richter D, et al. Connection between polymer molecular weight, density, chain dimensions, and melt viscoelastic properties. Macromolecules. 1994; 27:4639-4647.

[145] Marvin RS. The linear viscoelastic behavior of rubberlike polymers and its molecular interpretation. In: Bergen JT, editor. Viscoelasticity: Phenomenological Aspects. New York: Academic Press; 1960. p. 27-54.

[146] Ferry JD. Viscoelastic properties of polymers. 3rd ed. New York: John Wiley \& Sons, Inc.; 1980.

[147] Benallal A, Marin G, Montfort J, et al. Linear viscoelasticity revisited - the relaxation function of monodisperse polymer melts. Macromolecules. 1993;26:7229-7235.

[148] Smith G, Paul W, Monkenbusch M, et al. Molecular dynamics of a 1,4-polybutadiene melt. Comparison of experiment and simulation. Macromolecules. 1999;32:8857-8865.

[149] Doi M. Explanation for the 3.4 power law of viscosity of polymeric liquids on the basis of the tube model. J Polym Sci Pt C-Polym Lett. 1981;19:265-273.

[150] Stephanou PS, Baig C, Tsolou G, et al. Quantifying chain reptation in entangled polymer melts: Topological and dynamical mapping of atomistic simulation results onto the tube model. J Chem Phys. 2010;132:124904.

[151] Stephanou PS, Mavrantzas VG. Quantitative predictions of the linear viscoelastic properties of entangled polyethylene and polybutadiene melts via modified versions of modern tube models on the basis of atomistic simulation data. J Non-Newton Fluid Mech. 2013; 200:111-130.

[152] Stephanou PS, Mavrantzas VG. Accurate prediction of the linear viscoelastic properties of highly entangled mono and bidisperse polymer melts. J Chem Phys. 2014;140:214903.

[153] Leygue A, Bailly C, Keunings R. A tube-based constitutive equation for polydisperse entangled linear polymers. J Non-Newton Fluid Mech. 2006;136:1-16.

[154] Leygue A, Bailly C, Keunings R. A differential tube-based model for predicting the linear viscoelastic moduli of polydisperse entangled linear polymers. J Non-Newton Fluid Mech. $2006 ; 133: 28-34$.

[155] Tang S, Greene MS, Liu WK. Two-scale mechanism-based theory of nonlinear viscoelasticity. J Mech Phys Solids. 2012;60:199-226.

[156] Hua CC, Schieber JD. Segment connectivity, chain-length breathing, segmental stretch, and constraint release in reptation models. I. Theory and single-step strain predictions. J Chem Phys. 1998;109:10018.

[157] Shanbhag S, Larson RG, Takimoto J, et al. Deviations from dynamic dilution in the terminal relaxation of star polymers. Phys Rev Lett. 2001;87:195502.

[158] Masubuchi Y, Takimoto J, Koyama K, et al. Brownian simulations of a network of reptating primitive chains. J Chem Phys. 2001;115:4387-4394.

[159] Doi M, Takimoto J. Molecular modelling of entanglement. Philos Trans R Soc A-Math Phys Eng Sci. 2003;361:641-650.

[160] Khaliullin RN, Schieber JD. Self-consistent modeling of constraint release in a singlechain mean-field slip-link model. Macromolecules. 2009;42:7504-7517. 
[161] Sgouros AP, Megariotis G, Theodorou DN. Slip-Spring Model for the Linear and Nonlinear Viscoelastic Properties of Molten Polyethylene Derived from Atomistic Simulations. Macromolecules. 2017;50:4524-4541.

[162] Chappa VC, Morse DC, Zippelius A, et al. Translationally invariant slip-spring model for entangled polymer dynamics. Phys Rev Lett. 2012;109:148302.

[163] Uneyama T, Masubuchi Y. Multi-chain slip-spring model for entangled polymer dynamics. J Chem Phys. 2012;137:154902.

[164] Langeloth M, Masubuchi Y, Böhm MC, et al. Recovering the reptation dynamics of polymer melts in dissipative particle dynamics simulations via slip-springs. J Chem Phys. 2013;138:104907.

[165] Ramírez-Hernández A, Detcheverry FA, Peters BL, et al. Dynamical simulations of coarse grain polymeric systems: rouse and entangled dynamics. Macromolecules. 2013; 46:6287-6299.

[166] Ramírez-Hernández A, Peters BL, Andreev M, et al. A multichain polymer slip-spring model with fluctuating number of entanglements for linear and nonlinear rheology. J Chem Phys. 2015;143:243147.

[167] Liu C, He J, Keunings R, et al. Do tube models yield consistent predictions for the relaxation time and apparent plateau modulus of entangled linear polymers? Macromolecules. 2006;39:3093-3097.

[168] Vogiatzis GG, Megariotis G, Theodorou DN. Equation of state based slip spring model for entangled polymer dynamics. Macromolecules. 2017;50:3004-3029.

[169] Nafar Sefiddashti MH, Edwards BJ, Khomami B. Individual chain dynamics of a polyethylene melt undergoing steady shear flow. J Rheol. 2015;59:119-153.

[170] Nafar Sefiddashti MH, Edwards BJ, Khomami B. Communication: A coil-stretch transition in planar elongational flow of an entangled polymeric melt. J Chem Phys. 2018; 148:141103.

[171] Dobson M. Periodic boundary conditions for long-time nonequilibrium molecular dynamics simulations of incompressible flows. J Chem Phys. 2014;141:184103.

[172] O'Connor TC, Alvarez NJ, Robbins MO. Relating chain conformations to extensional stress in entangled polymer melts. Phys Rev Lett. 2018;121:047801.

[173] Mead DW. Determination of molecular weight distributions of linear flexible polymers from linear viscoelastic material functions. J Rheol. 1994;38:1797-1827.

[174] Shanbhag S. Analytical rheology of polymer melts: state of the art. ISRN Materials Science. 2012;2012:732176.

[175] Larson RG. Materials science: Predicting the flow of real polymers. Science. 2011; 333:1834-1835.

[176] Snijkers F, Pasquino R, Olmsted PD, et al. Perspectives on the viscoelasticity and flow behavior of entangled linear and branched polymers. J Phys-Condes Matter. 2015; 27:473002.

[177] Read DJ, Auhl D, Das C, et al. Linking models of polymerization and dynamics to predict branched polymer structure and flow. Science. 2011;333:1871-1874.

[178] Tobita H. Simultaneous long-chain branching and random scission: I. Monte Carlo simulation. J Polym Sci Pt B-Polym Phys. 2001;39:391-403.

[179] Daniels PH. A brief overview of theories of PVC plasticization and methods used to evaluate PVC-plasticizer interaction. J Vinyl Addit Techn. 2009;15:219-223.

[180] Wypych G. Handbook of plasticizers. Toronto: ChemTec Publishing; 2004.

[181] Li D, Panchal K, Mafi R, et al. An atomistic evaluation of the compatibility and plasticization efficacy of phthalates in poly(vinyl chloride). Macromolecules. 2018;51:69977012 . 